This PDF is a selection from a published volume from the National Bureau of Economic Research

Volume Title: The Intended and Unintended Effects of U.S. Agricultural and Biotechnology Policies

Volume Author/Editor: Joshua S. Graff Zivin and Jeffrey M. Perloff, editors

Volume Publisher: University of Chicago Press

Volume ISBN: 0-226-98803-1; 978-0-226-98803-0 (cloth)

Volume URL: http://www.nber.org/books/perl10-1

Conference Date: March 4-5, 2010

Publication Date: February 2012

Chapter Title: Risk Response in Agriculture

Chapter Authors: Jeffrey LaFrance, Rulon Pope, Jesse Tack

Chapter URL: http://www.nber.org/chapters/c12108

Chapter pages in book: (p. 143 - 186) 


\title{
Risk Response in Agriculture
}

\author{
Jeffrey LaFrance, Rulon Pope, and Jesse Tack
}

\subsection{Introduction}

Farm and food policies affect crop acres, asset management, intensive and extensive margin decisions, and risk management choices in agricultural production. For example, in 1991, less than 25 percent of cropland ( 82 million acres) was covered by a federally subsidized crop insurance contract, with $\$ 11.2$ billion in total liability, $\$ 740$ million in insurance premiums, premium subsidies of 25 percent ( $\$ 190$ million) of gross farm premiums, and total indemnity payments of $\$ 955$ million. Relative to premiums paid by farmers ( $\$ 550$ million), for each $\$ 1.00$ in premiums paid by the typical insured farmer, $\$ 1.75$ in indemnity payments were received.

Even with this relatively profitable insurance program, farmer participation rates remained quite low. This outcome is likely due to the race to the bottom problem in a pooling equilibrium (LaFrance, Shimshack, and $\mathrm{Wu}$ 2000, 2001, 2002, 2004). However, Congress responded to the appearance of an incomplete insurance market with increased subsidies and many new forms of insurance.

The 1996 Federal Agricultural Improvement and Reform Act and the amendments to the 1938 Federal Crop Insurance Act that are commonly known as the Agricultural Risk Protection Act of 2000 mandated higher subsidy rates, the development and marketing of new insurance products for virtually every crop and livestock product produced in the United States, and

Jeffrey LaFrance is the Distinguished Professor of Agricultural and Resource Economics at Wahington State University, professor at the Paul G. Allen School for Global Animal Health at Washington State University, and professor of agricultural and resource economics at the University of California, Berkeley. Rulon Pope is professor of economics at Brigham Young University. Jesse Tack is assistant professor of agricultural economics at Mississippi State University. 
substantial subsidies for crop insurance marketing firms and large private reinsurance companies.

This change in farm policy greatly expanded the federal crop insurance program. In 2003, the Federal Crop Insurance Corporation (FCIC) provided insurance products for more than 100 crops on 217 million acres ( $2 / 3$ of all cropland). The total insurance liability was $\$ 40.6$ billion, with $\$ 3.4$ billion in insurance premiums, subsidies of almost 60 percent of gross premiums ( $\$ 2.0$ billion), and total indemnity payments of $\$ 3.2$ billion. The current program includes subsidy payments to private companies marketing federal crop insurance equal to 24.5 percent of gross premiums for administration and oversight ( $\mathrm{A} \& \mathrm{O})$, and to private reinsurance companies equal to 13.6 percent of gross premiums. Reinsurance companies also have the right to sell up to 50 percent of their contracts back to the FCIC (that is, to the taxpayer) at cost. The FCIC's Risk Management Agency's (RMA) book of business shows that 20 percent of the insured farms account for nearly 80 percent of indemnity payments. This suggests substantial adverse selection, as well as moral hazard, because the majority of the federally subsidized crop insurance products calculate premiums based on deviations from county-level yield trends. That is to say, FCIC insurance products are based on a pooling equilibrium established at the county level and, in some cases, larger areas known as risk regions.

The net effect is that for each $\$ 1.00$ in premiums actually paid by farmers, they receive an average of $\$ 2.40$ in indemnity payments, insurance marketing firms receive $\$ 0.40$ in $\mathrm{A} \& \mathrm{O}$ subsidies, and reinsurance companies make in the neighborhood of $\$ 0.45$ in profit due to the combined direct subsidies on premiums and their reinsurance rights with the FCIC, which allow them to cream, or high grade, the insurance pool.

In 2004, the RMA issued a request for proposals to develop subsidized pasture and range insurance for 440 million acres of private, public, and Native American pasture and rangeland in the country. Many agricultural economists at land grant universities across the country actively consult with the RMA and private insurance companies to develop new and expand existing federally subsidized crop insurance products.

Although this is only one example of the ubiquitous nature of federal intervention in U.S. agriculture, there is a large literature on the impacts of subsidized crop insurance on variable input use and the intensive margin (Nelson and Loehman 1987; Chambers 1989; Quiggin 1992; Horowitz and Lichtenberg 1994; Smith and Goodwin 1996; Babcock and Hennessy 1996). The effects of subsidized crop insurance programs on the extensive margin also has been the subject of considerable analysis (Gardner and Kramer 1986; Goodwin and Smith 2004; Keeton, Skees, and Long 1999; and Young et al. 2000; LaFrance, Shimshack, and Wu 2000, 2001, 2002, 2004), all of which conclude that subsidized crop insurance results in additional planting of marginal crop acres. Williams (1988), Turvey (1992), Wu (1999), and 
Soule, Nimon, and Mullarkey (2000) examine the impacts of subsidized crop insurance on choices of crop mixes and acreage decisions. Empirical results in this component of the literature suggest that economically marginal land also is environmentally marginal. These results all suggest that subsidized crop insurance tends to increase environmental degradation. Even so, very little of the previous work in this area uses structural models or takes into account the dynamic nature of agricultural decision making under risk.

To better understand these and many other longstanding issues in U.S. agricultural policy, this chapter develops a comprehensive structural econometric model of variable input use; crop mix and acreage choices; investment and asset management decisions; and consumption, savings and wealth accumulation in a stochastic dynamic programming model of farm-level decision making over time. This model develops and establishes clear and intuitively appealing relationships between dynamic life-cycle consumption theory, the theory of the competitive firm subject to risk, and modern finance theory.

We present, discuss, and apply a new class of variable input demand systems in a multiproduct production setting. All of the models in this class can be estimated with observable data; are exactly aggregable; are consistent with economic theory for any von Neumann-Morgenstern expected utility function; and can be used to nest and test exact aggregation, economic regularity, functional form, and flexibility. Implications of monotonicity, concavity in prices, and convexity in outputs and quasi-fixed inputs are developed for a specific subset of this class of models. We then apply this to thirteen variable inputs in U.S. agriculture over the sample period 1960 to 1999.

The results obtained from this empirical variable cost model are used to help develop a structural model of the dynamic decision problems faced by a generic agricultural producer. In this life-cycle model of agricultural decisions under risk, farmers create income and wealth through savings, investment in risky financial assets, own-labor choices both on- and off-farm, and agricultural production and investment activities. This disciplines the economic theory of agricultural production over time and under risk and helps to better identify risk preferences and other model parameters.

While it is beyond the scope of this chapter, one could solve the system of arbitrage equations derived in section 5.4 for optimal farmland, capital, and share allocations and use the estimated parameters to simulate the effects of a variety of different agricultural policy instruments. For example, one could use the parameter estimates to investigate the effect of policies targeting lower food prices, taking into account the supply lag driven by partial adjustment of land and capital over time. In addition, one could use these estimates to investigate how far the extensive margin will expand or contract in response to a variety of policy scenarios including subsidization of corn for ethanol, an increase in the variety of subsidized crop insurance 
products, and the introduction of new revenue support programs such as the Average Crop Revenue Election (ACRE) program.

Our empirical findings have important implications for the agricultural economy and associated policy instruments. The majority of theoretical and empirical agricultural policy analysis assumes curvature of the farmagents utility function and the inability of these agents to invest in off-farm revenue opportunities when making on-farm production decisions. While our empirical findings do find evidence of utility function curvature, we also find that this function is much flatter than is typically assumed or estimated. Importantly, this finding suggests that previous studies of risk-reducing policy instruments have likely overstated on-farm impacts. We also find evidence that farmers respond to off-farm revenue opportunities, which has important implications for the current debate on the subsidization of corn for ethanol. Given the role of the general economy in providing farm-agents with off-farm revenue opportunities, this finding suggests that farm-level impacts of ethanol subsidization have been affected by the recent downturn in the economy. This is a subtle point that is not addressed in the literature surrounding this debate but implies that analysis conducted prior to the downturn is no longer relevant.

\subsection{The Production Model and Two Results}

Five longstanding questions in economics, econometrics, and agricultural economics are the choice of functional form; the degree of flexibility; the conditions required for and regions of economic regularity; consistency with aggregation from micro- to macro-level data; and how best to handle simultaneous equations bias, errors in variables, and latent variables in a structural econometric model. In this chapter, we attempt to deal with all of these issues in a coherent framework for the analysis of a life-cycle model of agricultural production, investment, consumption, and savings decisions.

Analysis of multiproduct behavior of firms is common in economics (Färe and Primont 1995; Just, Zilberman, and Hochman 1988; Shumway 1983; Lopez 1983; Akridge and Hertel 1986). A large literature on functional structure and duality guides empirical formulations and testing based on concepts of nonjointness and separability (Lau 1972, 1978; Blackorby, Primont, and Russell 1977, 1978; Chambers 1984). Nonjoint production processes reduce to additivity in costs (Hall 1973; Kohli 1983). Separability in a partition of inputs or outputs often results in separability in a similar partition of prices (Blackorby, Primont, and Russell 1977; Lau 1978).

The neoclassical model of conditional demands for variable inputs with joint production, quasi-fixed inputs, and production and output price risk is

$$
\boldsymbol{x}(\boldsymbol{w}, \overline{\boldsymbol{y}}, \boldsymbol{z})=\arg \min \left\{\boldsymbol{w}^{\top} \boldsymbol{x}: F(x, \overline{\boldsymbol{y}}, \boldsymbol{z}) \leq 0\right\}
$$


where $\boldsymbol{x} \in \mathscr{X} \subseteq \mathbb{R}_{+}^{n_{x}}$ is an $n_{x}$-vector of variable inputs, $\boldsymbol{w} \in \mathscr{W} \subseteq \mathbb{R}_{+}^{n_{x}}$ is an $n_{x}$-vector of variable input prices, $\overline{\boldsymbol{y}} \in \mathscr{Y} \subseteq \mathbb{R}_{+}^{n_{x}}$ is an $n_{y}$-vector of planned outputs, $\boldsymbol{z} \in \mathcal{Z} \subseteq \mathbb{R}_{+}^{n_{x}}$ is an $n_{z}$-vector of quasi-fixed inputs. ${ }^{1} F: \mathscr{X} \times \mathscr{Y} \times \mathcal{Z} \rightarrow \mathbb{R}$ is the joint production transformation function, which is the boundary of a closed and convex production possibilities set that is characterized by free disposal in inputs and outputs. Let the variable cost function be denoted by $c(\boldsymbol{w}, \overline{\boldsymbol{y}}, \boldsymbol{z}) \equiv \boldsymbol{w}^{\top} \boldsymbol{x}(\boldsymbol{w}, \overline{\boldsymbol{y}}, \boldsymbol{z})$. We assume throughout that the production process is subject to supply shocks of the general form

$$
\boldsymbol{y}=\overline{\boldsymbol{y}}+\boldsymbol{h}(\overline{\boldsymbol{y}}, \boldsymbol{z}, \varepsilon), E[\boldsymbol{h}(\overline{\boldsymbol{y}}, \boldsymbol{z}, \varepsilon) \mid \boldsymbol{x}, \overline{\boldsymbol{y}}, \boldsymbol{z}]=\mathbf{0} .
$$

In either a static or a dynamic setting, it is a simple matter to show that equation (1) is implied by equation (2) and the expected utility hypothesis for all von Newman-Morgenstern preferences (Pope and Chavas 1994; Ball et al. 2010).

Planned output is a vector of latent, unobservable variables in production with supply risk. Hence, to estimate the demand system in equation (1) directly, one must either identify and estimate the expectations formation process or address the errors in variables problem associated with using $y$ in place of $\bar{y}$ in the demand equations (Pope and Chavas 1994). One branch of the literature advocates specifying an ex ante cost function where planned output is replaced by cost, which is observable when the variable inputs are committed to the production process (Pope and Chavas 1994; Pope and Just 1996; Chambers and Quiggin 2000; Chavas 2008; Ball et al. 2010; LaFrance and Pope 2010). In a joint production process, this requires making assumptions such that the input demands are functions of input prices, the levels of quasi-fixed inputs, and the variable cost of production,

$$
\boldsymbol{x}(\boldsymbol{w}, \overline{\boldsymbol{y}}, \boldsymbol{z})=\tilde{\boldsymbol{x}}[\boldsymbol{w}, \boldsymbol{z}, c(\boldsymbol{w}, \overline{\boldsymbol{y}}, \boldsymbol{z})] .
$$

This approach makes particular sense in agriculture where outputs and output prices are observed ex post. The main result of LaFrance and Pope (2010) on this question is as follows (a proof of this result is presented in appendix A of this chapter).

Proposition 1. The following functional structures are equivalent:

$$
\begin{aligned}
x(w, \bar{y}, z) & \equiv \tilde{x}[w, c(w, \bar{y}, z), z] \\
c(w, \bar{y}, z) & \equiv \tilde{c}[w, z, \theta(\bar{y}, z)] \\
F(x, \bar{y}, z) & \equiv \tilde{F}[\boldsymbol{x}, \boldsymbol{z}, \theta(\bar{y}, \boldsymbol{z})]
\end{aligned}
$$

1. In this section, we use $\overline{\boldsymbol{y}} \in \mathbb{R}_{+}^{n_{y}}$ to denote the $n_{y}$-vector of planned or expected outputs to simplify notation. In later sections, we modify this notation to $\overline{\boldsymbol{Y}}=\boldsymbol{a} \cdot \overline{\boldsymbol{y}}$, where $\boldsymbol{a}$ is the $n_{y}$-vector of acres planted to crops, $\overline{\boldsymbol{y}}$ now is the $n_{y}$-vector of expected yields, and $\bullet$ is the Hadamard product. We also define $z$ explicitly in the following. 
In other words, outputs must be weakly separable from the variable input prices in the variable cost function. This, in turn, is equivalent to outputs being weakly separable from the variable inputs in the joint production transformation function.

This is a tight result - separability is both necessary and sufficient for the variable inputs to be estimable in ex ante form. Hereafter, we will call any such demand model an ex ante joint production system.

A second common issue in the empirical analysis of agricultural supply decisions is that some level of aggregation is virtually unavoidable. Microlevel data needed to study input use, acreage allocations, and asset management choices at the farm level do not exist. Aggregation from micro-level decision makers to macro-level data has been studied extensively in consumer theory. ${ }^{2}$ This has received less attention in production economics (Chambers and Pope 1991, 1994; Ball et al. 2010; LaFrance and Pope 2008, 2010).

Recently, LaFrance and Pope (2009) obtained the indirect preferences for all exactly aggregable, full rank systems of consumer demand equations. Their result extends directly to production in the following way. Let $K \in$ $\{1,2,3,4\}$ and define the smooth real-valued function, $\omega: \mathbb{R} \times \mathbb{R} \rightarrow \mathbb{R}$, by

(7) $\omega[\eta(w), \theta]= \begin{cases}\theta, & \text { if } K=1,2 \text { or } K=3 \text { and } \lambda^{\prime}(s)=0, \\ \theta+\int_{0}^{\eta(w)}\left[\lambda(s)+\omega(s, \theta)^{2}\right] d s, & \text { if } K=3,4 \text {, and } \lambda^{\prime}(s) \neq 0,\end{cases}$

subject to $\omega(0, \theta)=\theta$ and $\partial \omega(0, \theta) / \partial s=\lambda(0)+\theta^{2}$, where $\eta: \mathscr{W} \rightarrow \mathbb{R}$ and $\lambda: \mathbb{R} \rightarrow \mathbb{R}$ are smooth, real-valued functions, and $\eta$ is $0^{\circ}$ homogeneous. A class of full rank and exactly aggregable ex ante production systems can be characterized as follows. ${ }^{3}$

Proposition 2. Let $\pi: \mathscr{W} \rightarrow \mathbb{R}_{++}, \pi \in \mathscr{C}^{\infty}$, be strictly positive valued, increasing, concave, and $1^{\circ}$ homogeneous; let $\eta: \mathscr{W} \rightarrow \mathbb{R}_{+}, \eta \in \mathscr{C}^{\infty}$, be positive valued and $0^{\circ}$ homogeneous; let $\alpha, \beta, \gamma, \delta: \mathscr{W} \rightarrow \mathbb{C}=\{a+\mathrm{v} b, a, b \in \mathbb{R}\}$, $\alpha, \beta, \gamma, \delta \in \mathscr{C}^{\infty}$, be $0^{\circ}$ homogeneous and satisfy $\alpha \delta-\beta \gamma \equiv 1, \imath=\sqrt{-1}$; and let $f: \mathbb{R}_{++} \rightarrow \mathbb{C}, f \in \mathscr{C}^{\infty}$, and $f^{\prime} \neq 0$. Then the variable cost function for any full rank, exactly aggregable, ex ante joint production system is a special case of

2. An important subset of the literature on this topic includes Gorman $(1953,1961,1981)$; Muellbauer (1975, 1976); Howe, Pollak, and Wales (1979); Deaton and Muellbauer (1980); Jorgenson, Lau, and Stoker (1980, 1982); Russell (1983, 1996); Jorgenson and Slesnick (1984, 1987); Lewbel (1987, 1988, 1989, 1990, 1991, 2003); Jorgenson (1990); Diewert and Wales (1987, 1988); Blundell (1988); van Daal and Merkies (1989); Jerison (1993); Russell and Farris (1993, 1998); Banks, Blundell, and Lewbel (1997), LaFrance et al. (2002); LaFrance (2004); LaFrance, Beatty, and Pope (2006); and LaFrance and Pope (2009). The focus in the literature has been interior solutions and smooth demand equations. We remain faithful to this approach throughout the present chapter.

3. This result is consistent with exact aggregation as defined by Gorman (1981). One part of our ongoing work is to extend this class to Lau's (1982) definition of exact aggregation, generalizing the left-hand side of equation (8) to $f[c(\boldsymbol{w}, \overline{\boldsymbol{y}}, \boldsymbol{z}) / \pi, \boldsymbol{z}]$, wherein cost and quasi-fixed inputs vary across individual economic agents. 


$$
f\left(\frac{c(\boldsymbol{w}, \bar{y}, \boldsymbol{z})}{\pi(\boldsymbol{w})}\right)=\frac{\alpha(\boldsymbol{w}) \omega[\eta(w), \theta(\bar{y}, \boldsymbol{z})]+\beta(\boldsymbol{w})}{\gamma(\boldsymbol{w}) \omega[\eta(\boldsymbol{w}), \theta(\overline{\boldsymbol{y}}, \boldsymbol{x})]+\delta(\boldsymbol{w})}
$$

LaFrance and Pope (2009) present a complete proof of necessity in the case of consumer choice theory. Their proof applies to the current problem with only minor changes in notation. Sufficiency is shown here by considering the structure of the input demands generated by equation (8). This is accomplished simply enough by differentiating with respect to $w$ and applying Shephard's lemma. To make the notation as compact as possible, let a bold subscript $\boldsymbol{w}$ denote a vector of partial derivatives with respect to the variable input prices and suppress the arguments of the functions $\{\alpha, \beta, \gamma, \delta$, $\eta, \pi\}$ to yield (after a large amount of straightforward but tedious algebra, which is presented in appendix B):

$$
\begin{aligned}
\boldsymbol{x}=\frac{\pi_{w}}{\pi} c+\pi & \left\{\left[\alpha \beta_{w}-\beta \alpha_{w}+\left(\alpha^{2} \lambda+\beta^{2}\right) \eta_{w}\right] \frac{1}{f^{\prime}}\right. \\
& -\left[\alpha \delta_{w}-\delta \alpha_{w}+\gamma \beta_{w}-\beta \gamma_{w}+2(\alpha \gamma \lambda+\beta \delta) \eta_{w}\right] \frac{f}{f^{\prime}} \\
& \left.+\left[\gamma \delta_{w}-\delta \gamma_{w}+\left(\gamma^{2} \lambda+\delta^{2}\right) \eta_{w}\right] \frac{f^{2}}{f^{\prime}}\right\} .
\end{aligned}
$$

Thus, equation (8) generates input demands that have the finitely additive and multiplicatively separable structure of any full rank, exactly aggregable system (Gorman 1981; Lau 1982; Lewbel 1989). Note that there are potentially up to four linearly independent variable cost terms on the right with four associated linearly independent vectors of input price functions. Hence, any system generated by equation (8) will have rank up to, but no greater than four, the highest possible rank (Lewbel 1987, 1990, 1991; LaFrance and Pope 2009).

A third issue when estimating a system of variable input demand equations such as equation (9) is the fact that quasi-fixed inputs, planned outputs, variable input prices, and total variable cost all are jointly determined with the input demands. Consistent estimation under these conditions is addressed in the following empirical application.

\subsection{The Econometric Cost Model, Data, and Estimates}

Previous work at both state and national levels of aggregation with our data set strongly suggests that full rank three seriously overparameterizes the structural model for this data. As a result, we restrict attention here to a rank two model. In this part of the chapter, we analyze the conditional demands for thirteen variable inputs in U.S. agriculture: pesticides and herbicides; fertilizer; fuel and natural gas; electricity; purchased feed; purchased seed; purchased livestock; machinery repairs; building repairs; custom machinery services; veterinary services; other materials; and labor. The specification of the variable cost function normalized by the farm wage rate is, 


$$
\begin{aligned}
c_{t}\left(\tilde{\boldsymbol{w}}_{t}, A_{t}, K_{t}, \boldsymbol{a}_{t}, \overline{\boldsymbol{Y}}_{t}\right)= & {\left[\alpha_{10}+\boldsymbol{\alpha}_{1}^{\top} \tilde{\boldsymbol{w}}_{t}\right] A_{t}+\left[\alpha_{20}+\boldsymbol{\alpha}_{2}^{\top} \tilde{\boldsymbol{w}}_{t}\right] K_{t} } \\
& +\sqrt{\tilde{\boldsymbol{w}}_{t}^{\top} \boldsymbol{B}_{\tilde{\boldsymbol{w}}_{t}}+2 \boldsymbol{\gamma}^{\top} \tilde{\boldsymbol{w}}_{t}+1} \times \theta\left(A_{t}, K_{t}, \boldsymbol{a}_{t}, \overline{\boldsymbol{Y}}_{t}\right),
\end{aligned}
$$

where $\boldsymbol{z}_{t}=\left[A_{t} K_{t} \boldsymbol{a}_{t}\right]^{\top} ; A_{t}$ is farmland, $K_{t}$ is the value of farm capital; $\boldsymbol{a}_{t}=$ $\left[a_{1 t} a_{2 t} \ldots a_{n_{v}}\right]^{\top}$ is the $n_{y}$-vector of acres planted to crops; $A_{t}=a_{0 t}+\underline{\mathbf{\iota}}^{\top} \boldsymbol{a}_{t}$, with $a_{0 t}$ denoting farmland that is not devoted to crop production; $\overline{\boldsymbol{Y}}_{t}=$ $\left[a_{1 t} \bar{y}_{1 t} \ldots a_{n_{y} t} \bar{y}_{n_{y} t}\right]^{\top}$ is the $n_{y}$-vector of planned crop production, with each element defined as the product of acres planted to the crop times the expected yield per acre; and $\tilde{\boldsymbol{w}}_{t}=\left[w_{1 t} / w_{n_{x} t}, \ldots, w_{n_{x}-1 t} / w_{n_{x}}\right]^{\top}$ is the $\left(n_{x}-1\right)$-vector of variable input prices except the farm wage normalized by $w_{n_{x}}$.

We treat the $n_{\mathrm{x}}^{\text {th }}$ input, labor, asymmetrically with respect to the other inputs both in the structural and stochastic parts of the econometric model. To conserve and simplify notation from this point forward, we drop the tilde $(\sim)$ over the first $n_{x}-1$ input prices, absorb the normalization by $w_{n_{x}}$ into the notation for variable cost and the $n_{x}-1$ first input prices, and define $N=$ $n_{x}-1$.

We assume constant returns to scale so that $\theta\left(A_{t}, K_{t}, \boldsymbol{a}_{t}, \boldsymbol{Y}_{t}\right)$ is $1^{\circ}$ homogeneous. Define $\alpha_{1}\left(\boldsymbol{w}_{t}\right)=\alpha_{10}+\boldsymbol{\alpha}_{1}^{\top} \boldsymbol{w}_{t}, \alpha_{2}\left(\boldsymbol{w}_{t}\right)=\alpha_{20}+\boldsymbol{\alpha}_{2}^{\top} \boldsymbol{w}_{t}$, and $\beta\left(\boldsymbol{w}_{t}\right)=$ $\left(\boldsymbol{w}_{1}^{\top} \boldsymbol{B} \boldsymbol{w}_{t}+2 \boldsymbol{\gamma}^{\top} \boldsymbol{w}_{t}+1\right)^{1 / 2}$. The necessary and sufficient conditions for the variable cost function to be increasing and concave in the variable input prices throughout an open set containing the data points are as follows (see appen$\operatorname{dix} C$ for a complete derivation of the cost function and $\theta$ ):

Monotonicity in $w$ :

$$
\begin{aligned}
\frac{\partial c\left(\boldsymbol{w}_{t}, A_{t}, K_{t}, \boldsymbol{a}_{t}, \overline{\boldsymbol{Y}}_{t}\right)}{\partial \boldsymbol{w}} & =\boldsymbol{\alpha}_{1} A_{t}+\boldsymbol{\alpha}_{2} K_{t}+\frac{\theta}{\beta\left(\boldsymbol{w}_{t}\right)}\left(\boldsymbol{B} \boldsymbol{w}_{t}+\boldsymbol{\gamma}\right) \\
& =\boldsymbol{\alpha}_{1} A_{t}+\boldsymbol{\alpha}_{2} K_{t}+\left[\frac{c_{t}-\alpha_{1}\left(\boldsymbol{w}_{t}\right) A_{1}-\alpha_{2}\left(\boldsymbol{w}_{t}\right) K_{t}}{\boldsymbol{w}_{t}^{\top} \boldsymbol{B} \boldsymbol{w}_{t}+2 \boldsymbol{\gamma}^{\top} \boldsymbol{w}_{t}+1}\right]\left(\boldsymbol{B} \boldsymbol{w}_{t}+\boldsymbol{\gamma}\right) \geq 0 ;
\end{aligned}
$$

Concavity in $\boldsymbol{w}$ :

$$
\begin{aligned}
\frac{\partial^{2} c\left(\boldsymbol{w}_{t}, A_{t}, K_{t}, \boldsymbol{a}_{t}, \overline{\boldsymbol{Y}}_{t}\right)}{\partial \boldsymbol{w} \partial \boldsymbol{w}^{\top}}= & \frac{\theta}{\beta\left(\boldsymbol{w}_{t}\right)} \boldsymbol{B}-\frac{\theta}{\beta\left(\boldsymbol{w}_{t}\right)^{2}}(\boldsymbol{B} \boldsymbol{w}+\boldsymbol{\gamma})(\boldsymbol{B} \boldsymbol{w}+\boldsymbol{\gamma})^{\top} \\
= & {\left[\frac{c\left(\boldsymbol{w}_{t}, A_{t}, K_{t}, \boldsymbol{a}_{t}, \overline{\boldsymbol{Y}}_{t}\right)-\alpha_{1}\left(\boldsymbol{w}_{t}\right) A_{t}-\alpha_{2}\left(\boldsymbol{w}_{t}\right) K_{t}}{\boldsymbol{w}_{t}^{\top} \boldsymbol{B} \boldsymbol{w}_{t}+2 \boldsymbol{\gamma}^{\top} \boldsymbol{w}_{t}+1}\right.} \\
& \times\left[\boldsymbol{B}-\frac{\left(\boldsymbol{B} \boldsymbol{w}_{t}+\boldsymbol{\gamma}\right)\left(\boldsymbol{B} \boldsymbol{w}_{t}+\boldsymbol{\gamma}\right)^{\top}}{\left(\boldsymbol{w}_{t}^{\top} \boldsymbol{B} \boldsymbol{w}_{t}+2 \boldsymbol{\gamma}^{\top} \boldsymbol{w}_{t}+1\right)}\right]
\end{aligned}
$$

symmetric, negative semidefinite. Setting $\boldsymbol{B}=\boldsymbol{L} \boldsymbol{L}^{\top}+\boldsymbol{\gamma} \boldsymbol{\gamma}^{\top}$, where $\boldsymbol{L}$ is a (lower or upper) triangular matrix with nonzero main diagonal elements implies 


$$
\left[\begin{array}{ll}
\boldsymbol{B} & \boldsymbol{\gamma} \\
\boldsymbol{\gamma}^{\top} & 1
\end{array}\right]=\left[\begin{array}{ll}
\boldsymbol{L} & \boldsymbol{\gamma} \\
0^{\top} & 1
\end{array}\right]\left[\begin{array}{ll}
\boldsymbol{L}^{\top} & 0^{\top} \\
\boldsymbol{\gamma}^{\top} & 1
\end{array}\right]=\left[\begin{array}{ll}
\boldsymbol{L} \boldsymbol{L}^{\top}+\boldsymbol{\gamma} \boldsymbol{\gamma}^{\top} & \boldsymbol{\gamma} \\
\boldsymbol{\gamma}^{\top} & 1
\end{array}\right]
$$

is positive definite. It follows that $\left[\boldsymbol{B}-(\boldsymbol{B} \boldsymbol{w}+\boldsymbol{\gamma})(\boldsymbol{B} \boldsymbol{w}+\boldsymbol{\gamma})^{\top} /\left(\boldsymbol{w}_{t}^{\top} \boldsymbol{B} \boldsymbol{w}_{t}+2 \boldsymbol{\gamma}^{\top} \boldsymbol{w}_{t}+1\right)\right]$ is positive semidefinite and that

$$
\left[\begin{array}{ll}
\boldsymbol{w}_{t}^{\top} & 1
\end{array}\right]\left[\begin{array}{cc}
\boldsymbol{B} & \boldsymbol{\gamma} \\
\boldsymbol{\gamma}^{\top} & 1
\end{array}\right]\left[\begin{array}{c}
\boldsymbol{w}_{t} \\
1
\end{array}\right]=\boldsymbol{w}_{t}^{\top} \boldsymbol{B} \boldsymbol{w}_{t}+2 \boldsymbol{\gamma}^{\top} \boldsymbol{w}_{t}+1>0 \forall \boldsymbol{w}_{t} \in \mathbb{R}_{+}^{n_{x}-1} .
$$

Given this, the variable cost function is concave in $\boldsymbol{w}$ if and only if

$$
c_{t}\left(\tilde{\boldsymbol{w}}_{t}, A_{t}, K_{t}, a_{t}, \overline{\boldsymbol{Y}}_{t}\right)<\left[\alpha_{0}+\boldsymbol{\alpha}_{1}^{\top} \tilde{\boldsymbol{w}}_{t}\right] A_{t}+\left[\alpha_{2}+\boldsymbol{\alpha}_{2}^{\top} \tilde{\boldsymbol{w}}_{t}\right] K_{t}
$$

(LaFrance, Beatty, and Pope 2006). Hence, we impose $\boldsymbol{B}=\boldsymbol{L} \boldsymbol{L}^{\top}+\boldsymbol{\gamma} \boldsymbol{\gamma}^{\top}$ during estimation and check the monotonicity conditions in equation (11) at all data points once the model is estimated and find that they are satisfied. We develop the specification for $\theta\left(A_{t}, K_{t}, \boldsymbol{a}_{t}, \overline{\boldsymbol{Y}}_{t}\right)$ in the section on life-cycle consumption and investment decisions and appendix $\mathrm{C}$.

Applying Shephard's lemma to equation (10) and rearranging terms then gives the empirical variable input demand equations in normalized expenditures per dollar of capital as

$$
\boldsymbol{e}_{t}=\boldsymbol{W}_{t}\left[\boldsymbol{\alpha}_{1} \frac{A_{t}}{K_{t}}+\boldsymbol{\alpha}_{2}+\left(\frac{\left(c_{t} / K_{t}\right)-\alpha_{1}\left(\boldsymbol{w}_{t}\right)\left(A_{2} / K_{t}\right)-\alpha_{2}\left(\boldsymbol{w}_{t}\right)}{\boldsymbol{w}_{t}^{\top} \boldsymbol{B} \boldsymbol{w}_{t}+2 \boldsymbol{\gamma}^{\top} \boldsymbol{w}_{t}+1}\right)\left(\boldsymbol{B} \boldsymbol{w}_{t}+\boldsymbol{\gamma}\right)\right]+\boldsymbol{u}_{t},
$$

where $\boldsymbol{W}_{t}=\operatorname{diag}\left[w_{i t}\right]$ is the diagonal matrix with $w_{i, t}$ as the $i^{\text {th }}$ main diagonal element, and $\boldsymbol{e}_{t}=\left[w_{1, t} x_{1, t} \ldots w_{n_{x}-1, t} x_{n_{x}-1, t}\right]^{\top}$ is the $\left(n_{x}-1\right)$-vector of normalized expenditures per dollar of capital on all inputs except labor, and we follow standard practice in the empirical analysis of demand systems and add a vector of random errors to the right-hand-side to obtain the empirical model. We assume that the errors terms for the twelve equations estimated follow to an unrestricted first-order autoregressive (AR[1]) process,

$$
\boldsymbol{u}_{t}=\boldsymbol{R} \boldsymbol{u}_{t-1}+\varepsilon_{t}, \varepsilon_{t} \text { i.i.d. }(0, \mathbf{\Sigma}), t=1, \ldots, T .
$$

As noted in the preceding section, we apply this model to annual aggregate data on thirteen variable inputs in U.S. agriculture (pesticides and herbicides, fertilizer, fuel and natural gas, electricity, purchased feed, purchased seed, purchased livestock, machinery repairs, building repairs, custom machinery services, veterinary services, other materials, and farm labor). The sample period is 1960 to 1999 . These data were compiled by the United States Department of Agriculture's (USDA) Economic Research Service (ERS) and is described in detail in Ball, Hallahan, and Nehring (2004). Farmland, equipment, buildings, and structures are treated as quasi-fixed inputs. Hereafter, this data set is called the Ball data.

Due to the way that several variables are constructed in the Ball data, it is necessary to modify and augment this data for empirical implemen- 
tation. First, we define the replacement cost of owner-operator labor by the farm wage rate. This implies that the return to owner-operator labor in the Ball data due to management skill is treated as a part of the residual claimant's quasi-rent. Second, we use a direct measure of the value of capital obtained from the ERS rather than the measures constructed in the Ball data. Third, estimates of the price of farmland are taken from state-level surveys conducted by the National Agricultural Statistics Service (NASS), rather than the constructed measures in the Ball data. Finally, we adjust the measure of agricultural land. The Census of Agriculture has reported land in farms in four- to five-year intervals for 1954, 1959, 1964, 1969, 1974, 1978, 1982, 1987, 1992, 1997, 2002, and 2007. These are the total farmland numbers used in the sample years that match the census years. The ERS reports the harvested acres for all major crops by state and year since 1947. This data is used to adjust the farmland measures in the Ball data as follows. First, the difference between total farmland in the Ball data and harvested acres is calculated for each noncensus year by state. Second, in each period between adjacent censuses, the average of this difference is calculated. This mean difference is treated as fixed in each of the three- or four-year intervals between census years and added to harvested acres to obtain the measure of farmland used in this study in those years of our sample period. We normalize costs, expenditures, and acres by capital rather than total land because we are more confident in the capital measure, and Pope, LaFrance, and Just (2007) have shown that deflating by a variable that is subject to measurement error leads to difficult econometric issues.

Estimation is by nonlinear generalized method of moments (GMM), which assumes a parametric $12 \times 12 \mathrm{AR}(1)$ process for the time series component and White/Huber robust covariance matrix estimator that is consistent under heteroskedasticity of an unknown form. The instruments are variable cost per unit of capital, land per unit of capital, and variable input prices all lagged two periods, plus the following general economy variables lagged one period: real per capita disposable personal income; unemployment rate; the real rate of return on AAA corporate thirty-year bonds; real manufacturing wage rate; real index of prices paid by manufacturers for materials and components; and real index of prices paid by manufacturers for fuel, energy and power. Per capita disposable personal income is deflated by the Consumer Price Index (CPI) for all items. The aggregate wholesale price variables are deflated by the implicit price deflator for gross domestic product (GDP). The real rate of return on corporate bonds is calculated as the nominal rate of return minus the midyear annual inflation rate.

Table 5.1 presents the estimated $12 \times 12$ AR(1) matrix. The Eigen values of the implied autocovariance structure are well within the stability region, with two real roots and five complex conjugate pairs: 


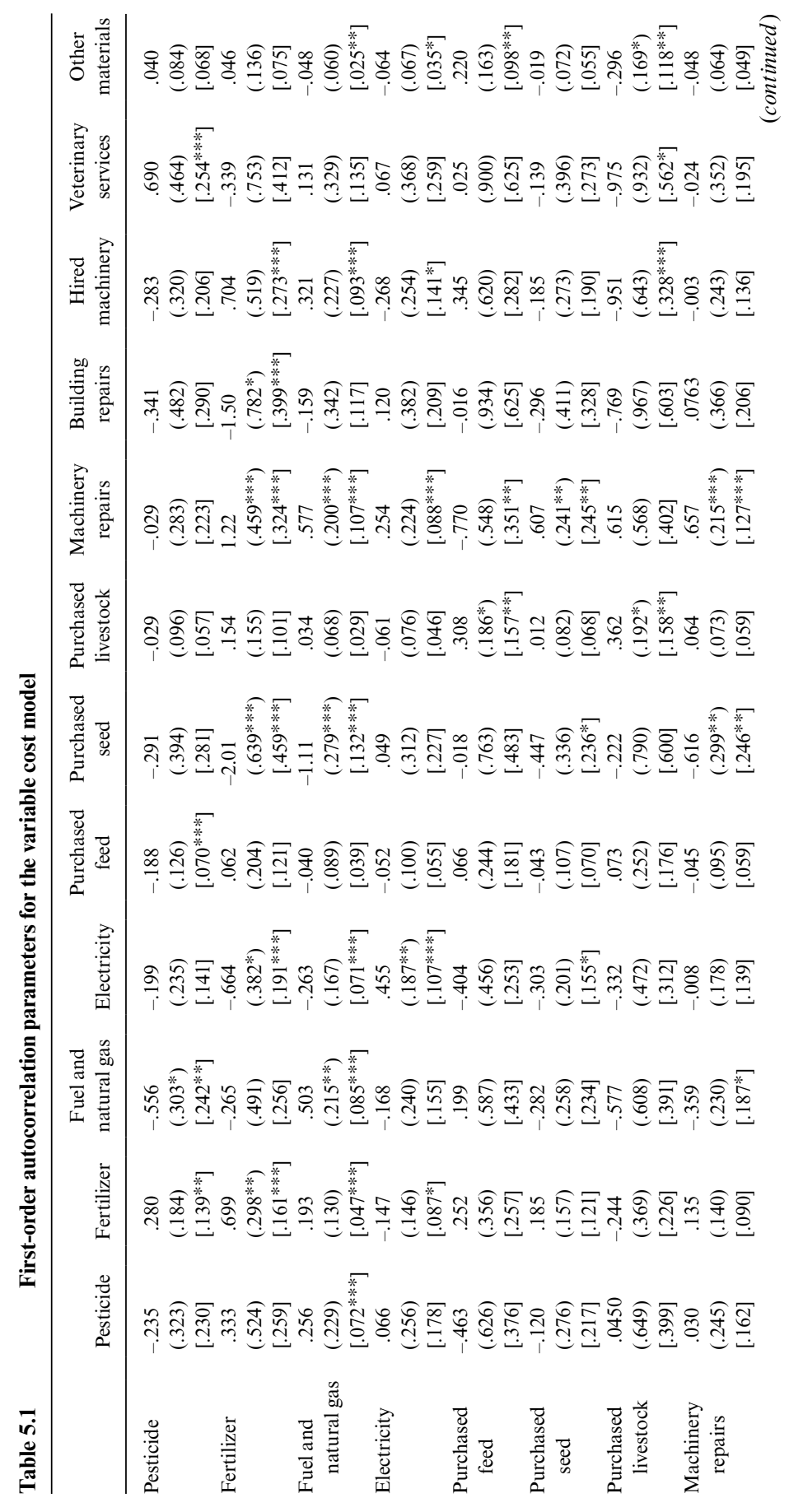




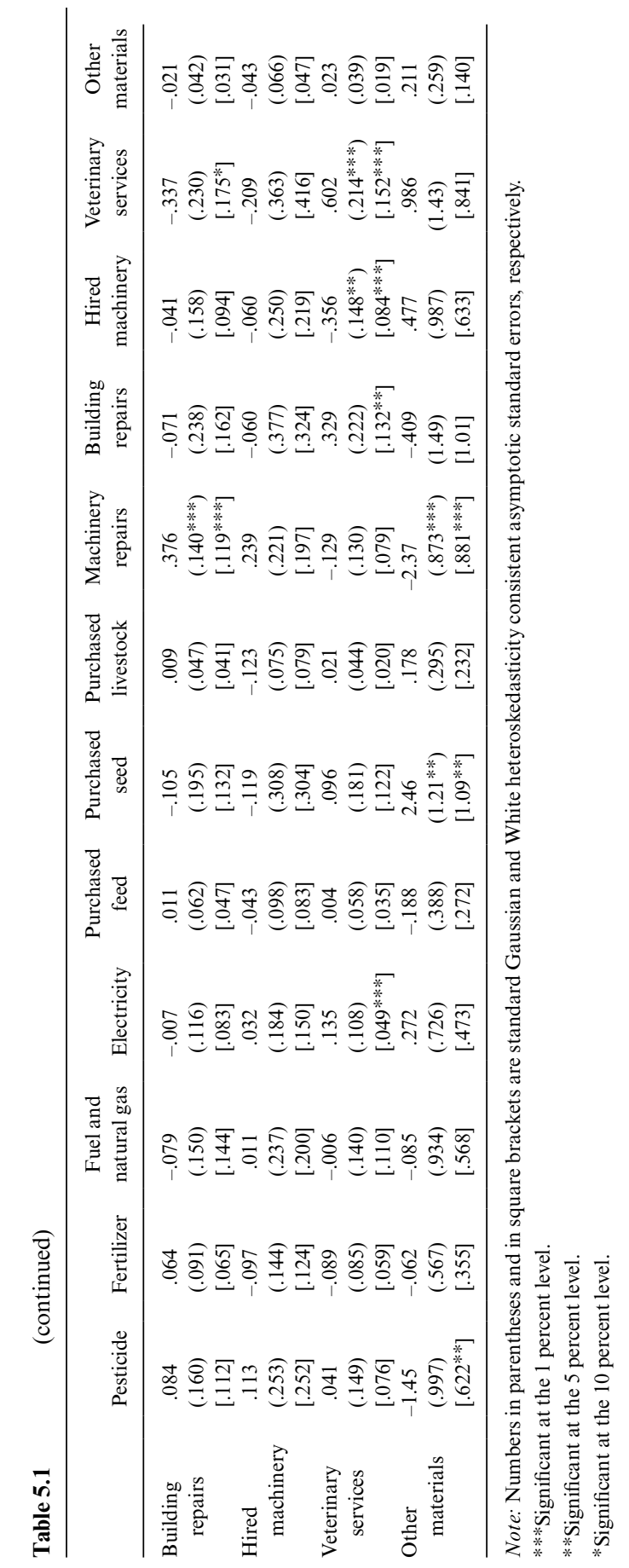




$$
\begin{aligned}
\lambda_{1} & =0.6772 \\
\lambda_{2} & =0.1294 \\
\lambda_{3,4} & =0.7594 \pm 0.29981, \text { modulus }=0.8165 \\
\lambda_{5,6} & =0.4104 \pm 0.52731, \text { modulus }=0.6682 ; \\
\lambda_{7,8} & =0.3056 \pm 0.33711, \text { modulus }=0.4550 ; \\
\lambda_{9,10} & =-0.4222 \pm 0.08321, \text { modulus }=0.4304 ; \\
\lambda_{11,12} & =-0.0863 \pm 0.26381, \text { modulus }=0.2776 .
\end{aligned}
$$

A system of twelve linear first-order difference equations has the same dynamic structure as a single twelfth-order linear difference equation. This implies that the time series properties of this model are quite complex. No evidence is found for any additional serial correlation in the data.

The single equation and systemwide first- and second-order Brownian bridge tests for specification error and parameter instability developed in LaFrance (2008) provide no evidence of misspecification or parameter instability. (Appendix D presents and discusses this set of within-sample residual test statistics.)

Table 5.2 presents the parameter estimates for the structural part of the model. To obtain a positive definite $\boldsymbol{B}$ matrix, the lower four main diagonal elements of the Choleski factor $\boldsymbol{L}$ were restricted to 0.01 , and the offdiagonal elements in the last four columns were restricted at 0.0 . In other words, the estimated symmetric but not curvature restricted $\boldsymbol{B}$ matrix has four negative Eigen values. As a consequence, the standard errors in table 5.2 are conditional on these inequality restrictions. The estimated structural parameters reported in table 5.2 generate a variable cost function that is increasing and weakly concave in all variable input prices throughout the data set. We conclude that this is a coherent and reasonable model of the short-run cost of production in U.S. agriculture.

\subsection{Crop Acres, Capital, Savings and Investment, and Consumption in Agriculture}

Although the organizational form of farms can vary widely, a recent report by Hoppe and Banker (2006) finds that 98 percent of U.S. farms remained family farms as of 2003. In a family farm, the entrepreneur controls the means of production and makes investment, consumption, and production decisions. In this section, we develop and analyze a model of the intertemporal nature of these decisions. The starting point is a model similar in spirit to Hansen and Singleton's (1983), but generalized to include consumption decisions and farm investments as well as financial investments and production decisions. The additional variable definitions required for this are as follows: 


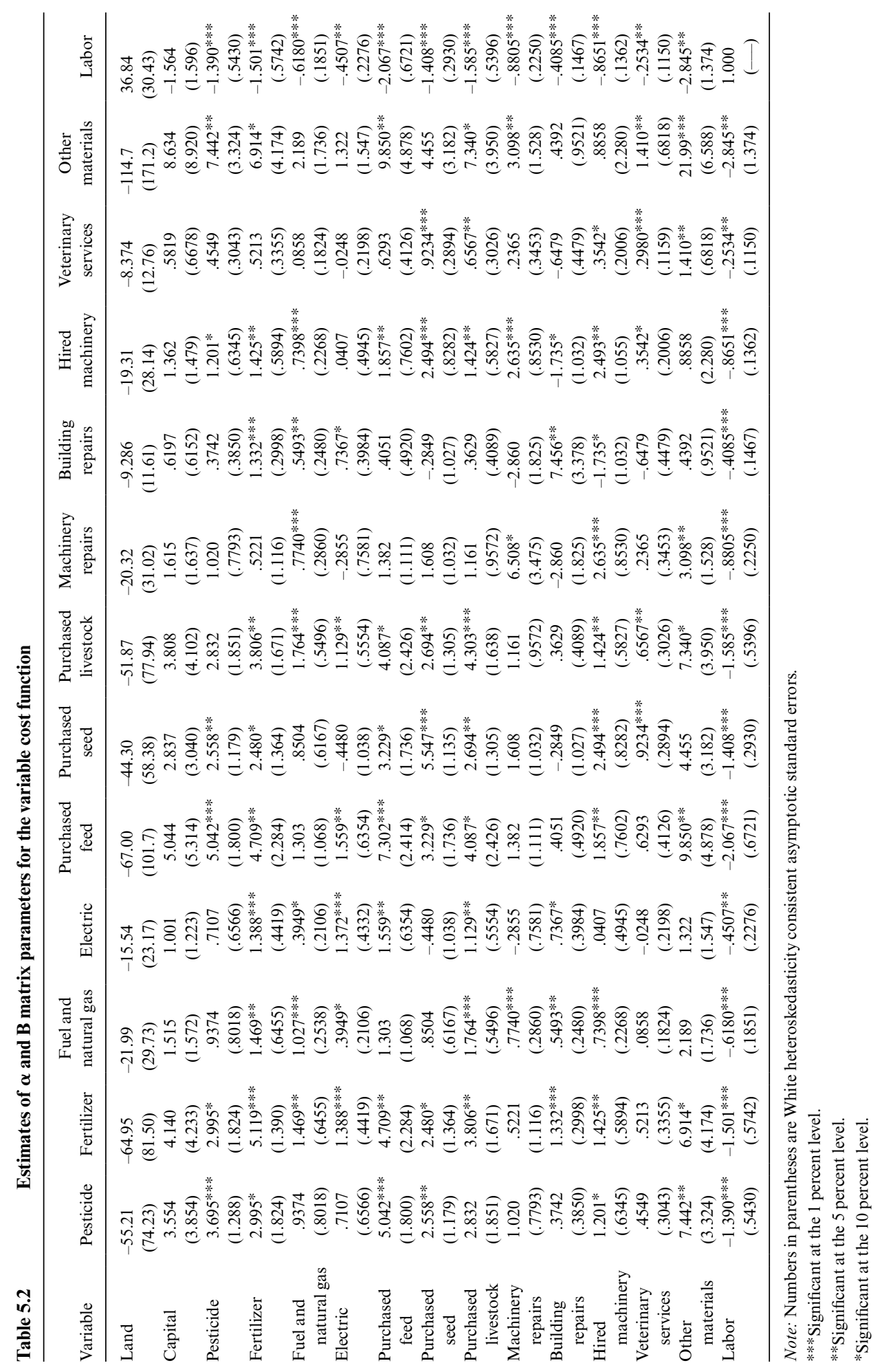


$W_{t}=$ beginning-of-period total wealth,

$b_{t}=$ current holding of bonds with a risk free rate of return $r_{t}$,

$f_{t}=$ current holding of a risky financial asset,

$p_{F, t}=$ beginning-of-period market price of the financial asset,

$\rho_{F, t+1}=$ dividend plus capital gains rate on the financial asset,

$a_{i, t}=$ current allocation of land to the $i^{\text {th }}$ crop, $i=1, \ldots, n_{Y}$,

$A_{t}=$ total quantity of farm land,

$p_{L, t}=$ beginning-of-period market price of land,

$\rho_{L, t+1}=\left(p_{L, t+1}-p_{L, t}\right) / p_{L, t}=$ capital gain rate on land,

$\bar{y}_{i, t}=$ expected yield per acre for the $i^{t h}$ crop, $i=1, \ldots, n_{Y}$,

$y_{i, t+1}=$ realized yield of the $i^{\text {th }}$ crop,

$p_{Y, t+1}=$ end-of-period realized market price for the $i^{\text {th }}$ farm product,

$\boldsymbol{q}_{t}=$ vector of quantities of consumption goods,

$\boldsymbol{p}_{Q, t}=$ vector of market prices for consumer goods,

$m_{t}=$ total consumption expenditures,

$u\left(\boldsymbol{q}_{t}\right)=$ periodic utility from consumption.

As with all discrete time models, timing can be represented in multiple ways. In the model used here, all financial returns and farm asset gains are assumed to be realized at the end of each time period (where depreciation is represented by a negative asset gain). Variable inputs are assumed to be committed to farm production activities at the beginning of each decision period, and the current period market prices for the variable inputs are known when these use decisions are made. Agricultural production per acre is realized stochastically at the end of the period such that

$$
y_{i, t+1}=\bar{y}_{i, t}\left(1+\varepsilon_{i, t+1}\right), i=1, \ldots, n_{Y},
$$

where $\varepsilon_{i, t+1}$ is a random output shock with $E\left(\varepsilon_{i, t+1}\right)=0$. Consumption decisions are made at the beginning of the decision period, and the current market prices of consumption goods are known when these purchases are made. Utility is assumed to be strictly increasing and concave in $\boldsymbol{q}_{t}$. The total beginning-of-period quantity of land is $A_{t}=\mathbf{\iota}^{\top} \boldsymbol{a}_{t}$, with $\mathbf{t}$ denoting an $n_{Y}$-vector of ones. Homogeneous land is assumed with a scalar price, $p_{L, t}$.

To simplify our derivations, we require an uncommon piece of matrix notation. The Hadamard/Schur product of two $n \times m$ matrices $\mathbf{A}$ and $\mathbf{B}$ is the matrix whose elements are element-by-element products of the elements of $\mathbf{A}$ and $\boldsymbol{B}, \boldsymbol{A} \cdot \boldsymbol{B}=\boldsymbol{C} \Leftrightarrow c_{i j}=a_{i j} b_{i j} \forall i, j$. This definition assists the derivation of the arbitrage conditions present in what follows.

Revenue at $t+1$ is the random price times production

$$
R_{t+1}=\sum_{i=1}^{n_{Y}}\left(p_{Y_{i}, t+1} \bar{Y}_{i, t} a_{i, t}\left(1+\varepsilon_{i, t+1}\right)\right) \equiv\left(\boldsymbol{p}_{Y, t+1} \bullet \boldsymbol{a}_{t} \cdot \overline{\boldsymbol{y}}_{t}\right)^{\top}\left(\mathbf{c}+\boldsymbol{\varepsilon}_{t+1}\right) .
$$

Wealth is allocated at the beginning of period $t$ to investments, the variable cost of production, and consumption,

$$
W_{t}=b_{t}+f_{t}+p_{L, t} A_{t}+K_{t}+c_{t}\left(\boldsymbol{w}_{t}, \boldsymbol{a}_{t}, K_{t}, \overline{\boldsymbol{Y}}_{t}\right)+m_{t} .
$$


Although some costs occur at or near harvest (near $t+1$ ), we include all costs in (21) at time $t$ because they are incurred before revenues are received. Consumer utility maximization yields the indirect utility function conditioned on consumer good prices and consumption expenditure,

$$
v\left(\boldsymbol{p}_{Q, t}, m_{t}\right) \equiv \max _{q \in R_{+}^{n_{Q}}}\left\{u(\boldsymbol{q}): \boldsymbol{p}_{Q, t}^{\top} \boldsymbol{q}=m_{t}\right\} .
$$

Realized end of period wealth is

$$
\begin{aligned}
W_{t+1}= & (1+r) b_{t}+\left(1+\rho_{F, t+1}\right) f_{t}+\left(1+\rho_{L, t+1}\right) p_{L, t} A_{t} \\
& +\left(1+\rho_{K, t+1}\right) K_{t}+\left(\boldsymbol{p}_{Y, t+1} \cdot \boldsymbol{a}_{t} \cdot \overline{\boldsymbol{y}}_{t}\right)^{\top}\left(\mathbf{\imath}+\boldsymbol{\varepsilon}_{t+1}\right),
\end{aligned}
$$

where $\rho_{K, t+1}$ is the proportional change in the value of capital held at the beginning of the production period. Thus, the decision maker's wealth is increased by net returns on assets and farm revenue. The owner/operator decision maker's intertemporal utility function is assumed to be

$$
U_{t}\left(\boldsymbol{q}_{1}, \ldots, \boldsymbol{q}_{T}\right)=\sum_{t=0}^{T}(1+r)^{-t} u\left(\boldsymbol{q}_{t}\right) .
$$

The producer is assumed to maximize von Neumann-Morgenstern expected utility of the discounted present value of the periodic utility flows from goods consumption.

By Euler's theorem, constant returns to scale implies linear homogeneity of the variable cost function in capital, land, and output. For the variable cost function derived and estimated in this chapter, this implies

$$
\begin{aligned}
c_{t}\left(\boldsymbol{w}_{t}, \boldsymbol{a}_{t}, A_{t}, K_{t}, \overline{\boldsymbol{Y}}_{t}\right) \equiv & \frac{\partial c_{t}\left(\boldsymbol{w}_{t}, \boldsymbol{a}_{t}, A_{t}, K_{t}, \overline{\boldsymbol{Y}}_{t}\right)}{\partial \boldsymbol{a}_{t}^{\top}} \boldsymbol{a}_{t}+\frac{\partial c_{t}\left(\boldsymbol{w}_{t}, \boldsymbol{a}_{t}, A_{t}, K_{t}, \overline{\boldsymbol{Y}}_{t}\right)}{\partial A_{t}} A_{t} \\
& +\frac{\partial c_{t}\left(\boldsymbol{w}_{t}, \boldsymbol{a}_{t}, A_{t}, K_{t}, \overline{\boldsymbol{Y}}_{t}\right)}{\partial K_{t}} K_{t}+\frac{\partial c_{t}\left(\boldsymbol{w}_{t}, \boldsymbol{a}_{t}, A_{t}, K_{t}, \overline{\boldsymbol{Y}}_{t}\right)}{\partial \overline{\boldsymbol{Y}}_{t}^{\top}} \overline{\boldsymbol{Y}}_{t} .
\end{aligned}
$$

The vector of expected crop outputs satisfies

$$
\overline{\boldsymbol{Y}}_{t}=\overline{\boldsymbol{y}}_{t} \boldsymbol{a}_{t},
$$

where $\bar{y}_{j, t}$ is the expected yield per acre, and $a_{j, t}$ is the number of acres planted for the $j^{\text {th }}$ crop. The variable cost function might depend on time due to technological change or other dynamic forces, and the subscript $t$ indicates this possibility. To distinguish quasi-fixed from variable inputs and to account for the possibility of hysteresis in agricultural investments, we allow for adjustment costs for total farmland and capital,

$$
C_{\mathrm{Adj}}\left(A_{t}-A_{t-1}, K_{t}-K_{t-1}\right)=\frac{1}{2} \gamma_{A}\left(A_{t}-A_{t-1}\right)^{2}+\frac{1}{2} \gamma_{K}\left(K_{t}-K_{t-1}\right)^{2},
$$

with $\gamma_{A}, \gamma_{K} \geq 0$.

This problem is solved by stochastic dynamic programming working 
backward recursively from the last period in the planning horizon to the first. In the last period, the optimal decision is to invest or produce nothing and consume all remaining wealth, that is, $m_{T}=W_{T}$. Denote the last period's optimal value function by $v_{T}\left(W_{T}, A_{T-1}, K_{T-1}\right)$. Then $v_{T}\left(W_{T}, A_{T-1}, K_{T-1}\right)=$ $v\left(\boldsymbol{p}_{Q, T}, W_{T}\right)$ is the optimal utility for the terminal period. For all other time periods, stochastic dynamic programming yields the Bellman backward recursion (Bellman and Dreyfus 1962). For an arbitrary $t<T$, the Lagrangean for the problem at time $t$ is

$$
\begin{aligned}
\boldsymbol{\ell}_{t}= & v\left(\boldsymbol{p}_{Q, t}, m_{t}\right)+(1+r)^{-1} E_{t}\left\{V _ { t + 1 } \left[(1+r) b_{t}+\left(1+\rho_{F, t+1}\right) f_{t}\right.\right. \\
& \left.\left.+p_{L, t+1} A_{t}+\left(1+\rho_{K, t+1}\right) K_{t}+\left(\boldsymbol{p}_{y, t+1} \bullet^{\boldsymbol{y}_{t}} \cdot \boldsymbol{a}_{t}\right)^{\top}\left(\mathbf{\imath}+\boldsymbol{\varepsilon}_{t+1}\right), A_{t}, K_{t}\right]\right\} \\
& +\lambda_{t}\left\{W_{t}-m_{t}-b_{t}-f_{t}-p_{L, t} A_{t}-K_{t}-c_{t}\left(\boldsymbol{w}_{t}, \boldsymbol{a}_{t}, A_{t}, K_{t}, \overline{\boldsymbol{y}}_{t} \boldsymbol{a}_{t}\right)\right. \\
& \left.-\frac{1}{2} \gamma_{A}\left(A_{t}-A_{t-1}\right)^{2}-\frac{1}{2} \gamma_{K}\left(K_{t}-K_{t-1}\right)^{2}\right\}+\mu_{t}\left(A_{t}-\mathbf{\iota}^{\top} \boldsymbol{a}_{t}\right),
\end{aligned}
$$

where $E_{t}(\bullet)$ is the conditional expectation at the beginning of period $t$ given information available at that point in time, $\lambda_{t}$ is the shadow price for the beginning-of-period wealth allocation constraint, and $\mu_{t}$ is the shadow price for the land allocation constraint. The first-order, necessary and sufficient Kuhn-Tucker conditions are the two constraints and the following:

$$
\begin{aligned}
\frac{\partial \ell_{t}}{\partial m_{t}}= & \frac{\partial v_{t}}{\partial m_{t}}-\lambda_{t} \leq 0, m_{t} \geq 0, m_{t} \frac{\partial \ell_{t}}{\partial m_{t}}=0 \\
\frac{\partial \ell_{t}}{\partial b_{t}}= & E_{t}\left(\frac{\partial V_{t+1}}{\partial W_{t+1}}\right)-\lambda_{t} \leq 0, b_{t} \geq, b_{t} \frac{\partial \ell_{t}}{\partial b_{t}}=0 \\
\frac{\partial \ell_{t}}{\partial f_{t}}= & (1+r)^{-1} E_{t}\left[\frac{\partial V_{t+1}}{\partial W_{t+1}}\left(1+\rho_{F, t+1}\right)\right]-\lambda_{t} \leq 0, f_{t} \geq 0, f_{t} \frac{\partial \ell_{t}}{\partial f_{t}}=0 \\
\frac{\partial \ell_{t}}{\partial A_{t}}= & (1+r)^{-1} E_{t}\left(\frac{\partial V_{t+1}}{\partial W_{t+1}} p_{L, t+1}+\frac{\partial V_{t+1}}{\partial A_{t}}\right) \\
& -\lambda_{t}\left[p_{L, t}+\frac{\partial c_{t}}{\partial A_{t}}+\gamma_{A}\left(A_{t}-A_{t-1}\right)\right]+\mu_{t} \leq 0, A_{t} \geq 0, A_{t} \frac{\partial \ell_{t}}{\partial A_{t}}=0 \\
\frac{\partial \ell_{t}}{\partial K_{t}}= & (1+r)^{-1} E_{t}\left[\frac{\partial V_{t+1}}{\partial W_{t+1}}\left(1+\rho_{K, t+1}\right)+\frac{\partial V_{t+1}}{\partial K_{t+1}}\right] \\
& -\lambda_{t}\left[1+\frac{\partial c_{t}}{\partial K_{t}}+\gamma_{K}\left(K_{t}-K_{t-1}\right)\right] \leq 0, K_{t} \geq 0, K_{t} \frac{\partial \ell_{t}}{\partial K_{t}}=0
\end{aligned}
$$

$$
\begin{aligned}
\frac{\partial \ell_{t}}{\partial \boldsymbol{a}_{t}}= & (1+r)^{-1} E_{t}\left[\frac{\partial V_{t+1}}{\partial W_{t+1}}\left(\boldsymbol{p}_{Y, t+1} \cdot \overline{\boldsymbol{y}}_{t}\right) \bullet\left(\mathbf{l}+\boldsymbol{\varepsilon}_{t+1}\right)\right] \\
& -\lambda_{t}\left(\frac{\partial c_{t}}{\partial \boldsymbol{a}_{t}}+\frac{\partial c_{t}}{\partial \overline{\boldsymbol{Y}}_{t}} \cdot \overline{\boldsymbol{y}}_{t}\right)-\mu_{t} \mathbf{\iota} \leq \mathbf{0}, \boldsymbol{a}_{t} \geq \mathbf{0}, \boldsymbol{a}_{t}^{\top} \frac{\partial \ell_{t}}{\partial \boldsymbol{a}_{t}}=0
\end{aligned}
$$




$$
\begin{aligned}
\frac{\partial \ell_{t}}{\partial \overline{\boldsymbol{y}}_{t}}= & (1+r)^{-1} E_{t}\left[\frac{\partial V_{t+1}}{\partial W} \boldsymbol{p}_{Y, t+1} \cdot \boldsymbol{a}_{t} \cdot\left(\mathbf{l}+\boldsymbol{\varepsilon}_{t+1}\right)\right]-\lambda_{t} \frac{\partial c_{t}}{\partial \overline{\boldsymbol{Y}}_{t}} \cdot \boldsymbol{a}_{t} \leq \mathbf{0}, \\
& \overline{\boldsymbol{y}}_{t} \geq \mathbf{0}, \overline{\boldsymbol{y}}_{t}^{\top} \frac{\partial \ell_{t}}{\partial \overline{\boldsymbol{y}}_{t}}=0 .
\end{aligned}
$$

We also have the following implications of the envelope theorem:

$$
\begin{aligned}
\frac{\partial V_{t}}{\partial W_{t}} & =\lambda_{t} ; \\
\frac{\partial V_{t}}{\partial A_{t-1}} & =\lambda_{t} \gamma_{A}\left(A_{t}-A_{t-1}\right) ; \\
\frac{\partial V_{t}}{\partial K_{t-1}} & =\lambda_{t} \gamma_{K}\left(K_{t}-K_{t-1}\right) ;
\end{aligned}
$$

where the variables $\left\{\lambda_{t}, A_{t}, K_{t}\right\}$ are all evaluated at their optimal choices.

Combining the Kuhn-Tucker conditions with the results of the envelope theorem and assuming an interior solution for consumption, bonds, and risky financial assets, we obtain the standard Euler equations for smoothing the marginal utility of consumption and wealth,

$$
\frac{\partial v_{t}}{\partial m_{t}}=E_{t}\left(\frac{\partial v_{t+1}}{\partial m_{t+1}}\right)=\frac{\partial V_{t}}{\partial W_{t}}=E_{t}\left(\frac{\partial V_{t+1}}{\partial W_{t+1}}\right)=\lambda_{t}=E_{t}\left(\lambda_{t+1}\right),
$$

and the standard arbitrage condition for excess returns to risky financial assets,

$$
E_{t}\left[\left(\rho_{F, t+1}-r\right) \frac{\partial V_{t+1}}{\partial W_{t+1}}\right]=0 .
$$

The complementary slackness of the Kuhn-Tucker condition in equation (35) implies that for each crop we have the supply condition under risk,

$$
E_{t}\left\{\frac{\partial V_{t+1}}{\partial W_{t+1}}\left[p_{Y, t+1}-(1+r) \frac{\partial c_{t}}{\partial \bar{Y}_{i, t}}\right]\right\} \bar{Y}_{i, t}=0, i=1, \ldots, n_{y} .
$$

For each crop produced in positive quantity, this reduces to the well-known result that the conditional covariance between the marginal utility of future wealth and the difference between the ex post realized market price the marginal cost of production must vanish. The multiplicative factor $1+r$ is multiplied by ex ante marginal cost so that these two economic values are measured at a common point in time-in the present case at the end of the production period.

To obtain the arbitrage condition for the level of investment in agriculture, we combine the linear homogeneity property of the variable cost function in $\left(\boldsymbol{a}_{t}, A_{t}, K_{t}, \overline{\boldsymbol{Y}}_{t}\right)$ from equation (25) with complementary slackness in KuhnTucker conditions in equations (33) to (37), 


$$
0=\frac{\partial \ell_{t}}{\partial \boldsymbol{a}_{t}^{\top}} \boldsymbol{a}_{t}+\frac{\partial \ell_{t}}{\partial A_{t}} A_{t}+\frac{\partial \ell_{t}}{\partial K_{t}} K_{t}
$$

which, after considerable rearranging and combining of terms, gives

$$
\begin{aligned}
E_{t}\left(\frac { \partial V _ { t + 1 } } { \partial W _ { t + 1 } } \left\{s_{K, t}\left(\rho_{K, t+1}-r\right)+s_{L, t}\left(\rho_{L, t+1}-r\right)+\pi_{t+1}\right.\right. \\
+s_{K, t} \gamma_{K}\left(K_{t+1}-(2+r) K_{t}+(1+r) K_{t-1}\right) \\
\left.\left.+s_{A, t} \gamma_{A}\left[A_{t+1}-(2+r) A_{t}+(1+r) A_{t-1}\right]\right\}\right)=0,
\end{aligned}
$$

where $s_{K, t}=K_{t} /\left(p_{L, t} A_{t}+K_{t}\right)$ is capital's share of the value of the investment in agriculture in period $t, s_{L, t}=p_{L, t} A_{t} /\left(p_{L, t} A_{t}+K_{t}\right)$ is land's share of the value of the investment in agriculture in period $t, s_{A, t}=A_{t} /\left(p_{L, t} A_{t}+K_{t}\right)$ is the ratio of the quantity of land to the value of the investment in agriculture at the beginning of the production period, and

$$
\pi_{t+1}=\frac{R_{t+1}-(1+r) c_{t}}{p_{L, t} A_{t}+K_{t}}
$$

is the ex post net return to crop production over the variable cost of production relative to the ex ante value of agricultural investment so that it is measured as a rate of return to agricultural production. The first three terms inside of the square brackets of equation (41) represent the total sum of the excess returns to agriculture, including the rate of net return to crop production over variable costs. The last two terms in square brackets capture the effects of adjustment costs for farm capital and farmland. This has the standard one period ahead and one period behind second-order difference structure common to quadratic adjustment cost models in dynamic optimization problems.

To implement this system of Euler equations, we assume that the indirect utility function for consumption goods is a member of the certainty equivalent class,

$$
v\left(p_{Q t}, m_{t}\right)=\frac{m_{t}}{\pi_{C}\left(p_{Q t}\right)}-\frac{1}{2} \beta\left[\frac{m_{t}}{\pi_{C}\left(p_{Q t}\right)}\right]^{2},
$$

where $0 \leq \beta<\pi_{C}\left(\boldsymbol{p}_{Q t}\right) / m_{t} \forall t$ and $\pi_{C}\left(\boldsymbol{p}_{Q t}\right)$ is the CPI for all items. Then the marginal utility of money in each period is

$$
\lambda_{t}=\frac{1-\beta\left[m_{t} / \pi_{C}\left(p_{Q t}\right)\right]}{\pi_{C}\left(p_{Q t}\right)} .
$$

This allows us to identify the effects of risk aversion separately from those of adjustment costs and hysteresis in agricultural investment decisions. We assume that the preferences of agricultural producers are of the same class as all other individuals in the economy. This allows use of the observable variable per capita personal consumption expenditure, rather than the latent variable wealth, to model the empirical arbitrage equations. 


\subsubsection{Empirical Arbitrage Equations and Data}

Let $n \leq n_{y}$ be the number of crops included in the empirical model. The specification that we choose for $\partial c_{t} / \partial \bar{Y}_{i, t}$ is (see appendix $\mathrm{C}$ for a complete derivation),

$$
\begin{gathered}
\frac{\partial c_{t}}{\partial \bar{Y}_{i, t}}=\widehat{\beta\left(\boldsymbol{w}_{t}\right)}\left(\theta_{i}+\sum_{j=1}^{n_{x}} \theta_{i j} \frac{\bar{Y}_{j, t}}{K_{t}}\right), \text { with } \\
\widehat{\beta\left(\boldsymbol{w}_{t}\right)}=\sqrt{\tilde{\boldsymbol{w}}_{t}^{\top} \hat{\boldsymbol{B}}^{\tilde{\boldsymbol{w}}_{t}}+2 \hat{\boldsymbol{\gamma}}^{\top} \tilde{\boldsymbol{w}}_{t} w_{n_{x}, t}+w_{n_{x}, t}^{2}} .
\end{gathered}
$$

We use the estimated $\widehat{\beta\left(\boldsymbol{w}_{t}\right)}$ obtained from the ex ante variable input demand system, and $\tilde{w}_{t}=\left[w_{1, t} \ldots w_{n_{x}-1, t}\right]^{\top}$ is the vector of variable input prices other than the farm wage. ${ }^{4}$ The $n+3$ empirical arbitrage/Euler equations, therefore, are

(46) Consumption / Bonds:

$$
\beta\left(m_{t+1}-m_{t}\right)=u_{1, t+1},
$$

Risky Assets:

$$
\left(1-\beta m_{t+1}\right)\left(\rho_{F, t+1}-r\right)=u_{2, t+1},
$$

Crops:

$$
\begin{aligned}
& \left(1-\beta m_{t+1}\right)\left[p_{Y_{i}, t+1}-(1+r) \widehat{\beta\left(\boldsymbol{w}_{t}\right)}\left(\theta_{i}+\sum_{j=1}^{n} \frac{\theta_{i j} Y_{j, t+1}}{K_{t}}\right)\right]=u_{i, t+1}, \\
& i=3, \ldots, n+2,
\end{aligned}
$$

Agriculture:

$$
\begin{aligned}
(1- & \left.\beta m_{t+1}\right)\left\{s_{K, t}\left(\rho_{K, t+1}-r\right)+s_{L, t}\left(\rho_{L, t+1}-r\right)+\pi_{t+1}\right. \\
& +s_{A, t} \gamma_{A}\left(A_{t+1}-(2+r) A_{t}+(1+r) A_{t-1}\right) \\
& \left.+s_{K, t} \gamma_{K}\left[K_{t+1}-(2+r) K_{t}+(1+r) K_{t-1}\right]\right\}=u_{n+3, t+1} .
\end{aligned}
$$

The estimation method for this part of the modeling exercise again is nonlinear three-stage least squares/generalized method of moments (NL3SLS/GMM) with a parametric AR(1) correction for autocorrelation and White/Huber heteroskedasticity consistent estimated covariance matrix. We restrict the parameter matrix $\Theta=\left[\theta_{i j}\right]$ to be positive semidefinite by estimating it in Choleski factored form, $\boldsymbol{\Theta}=\boldsymbol{Q} \boldsymbol{Q}^{T}$, where $\boldsymbol{Q}$ is a lower triangular matrix. 


\subsubsection{Empirical Results}

We analyze acreage and supply decisions under risk for ten crops with the greatest value in the United States in 2006: soybeans, corn, cotton, hay, potatoes, rice, sugar beets, sugarcane, tobacco, and wheat. Crop revenues includes the value of government payments that is been imputed in the Ball data, to at least partially capture the effects of farm-level price, income, and other subsidy and stabilization programs on the distribution of realized farm revenues. The ten crops analyzed in this study account for 94 to 95 percent of total farm revenue from crop production and an even larger share of crop acreage. In addition to the ten crop production decisions under risk, we estimate Euler equations for the excess return to investing in agriculture, personal consumption expenditures, and the rate of return to stocks as measured by the Standard \& Poor 500 index.

To ensure a consistent definition of real values in this component of the model, we deflate all nominal prices, revenues, costs, and other values by that year's CPI for all items. We scale all aggregate economic data - for example, the total value of agricultural investment in U.S. agriculture-by the U.S. population to measure these variables all in per capita units. As noted in the preceding, real per capita personal consumption expenditures represents the Euler equation for the marginal utility of money over time.

Table 5.3 presents the unrestricted $13 \times 13$ AR(1) coefficient matrix. Similar to the variable cost function model, Eigen values of the implied autocovariance structure are well within the stability region, with five real roots and four complex conjugate pairs:

$\lambda_{1}=0.8960$;

$\lambda_{2}=-0.5510$;

$\lambda_{3}=0.2829$

$\lambda_{4}=0.1800$

$\lambda_{5}=-0.0471$

$\lambda_{6,7}=0.2966 \pm 0.60581$, modulus $=0.6745$;

$\lambda_{8,9}=0.5789 \pm 0.0674 \mathrm{l}$, modulus $=0.5828$;

$\lambda_{10,11}=-0.0048 \pm 0.5157 \mathrm{l}$, modulus $=0.5157$;

$\lambda_{12,13}=-0.4497 \pm 0.1827 \mathrm{l}$, modulus $=0.4854$.

Also similar to the properties of the cost function estimates, there is no evidence of any additional serial correlation in the error terms, and all of the systemwide and single equation Brownian bridge tests fail to reject the null hypothesis of no model specification errors or parameter instability at all standard levels of significance.

Table 5.4 presents the parameter estimates for the conditional mean components of the arbitrage model. To obtain a positive definite $\boldsymbol{\Theta}$ matrix, the lower four main diagonal elements of the Choleski factor $\boldsymbol{Q}$ were restricted to 0.01 , and the off-diagonal elements in the last four columns were restricted 


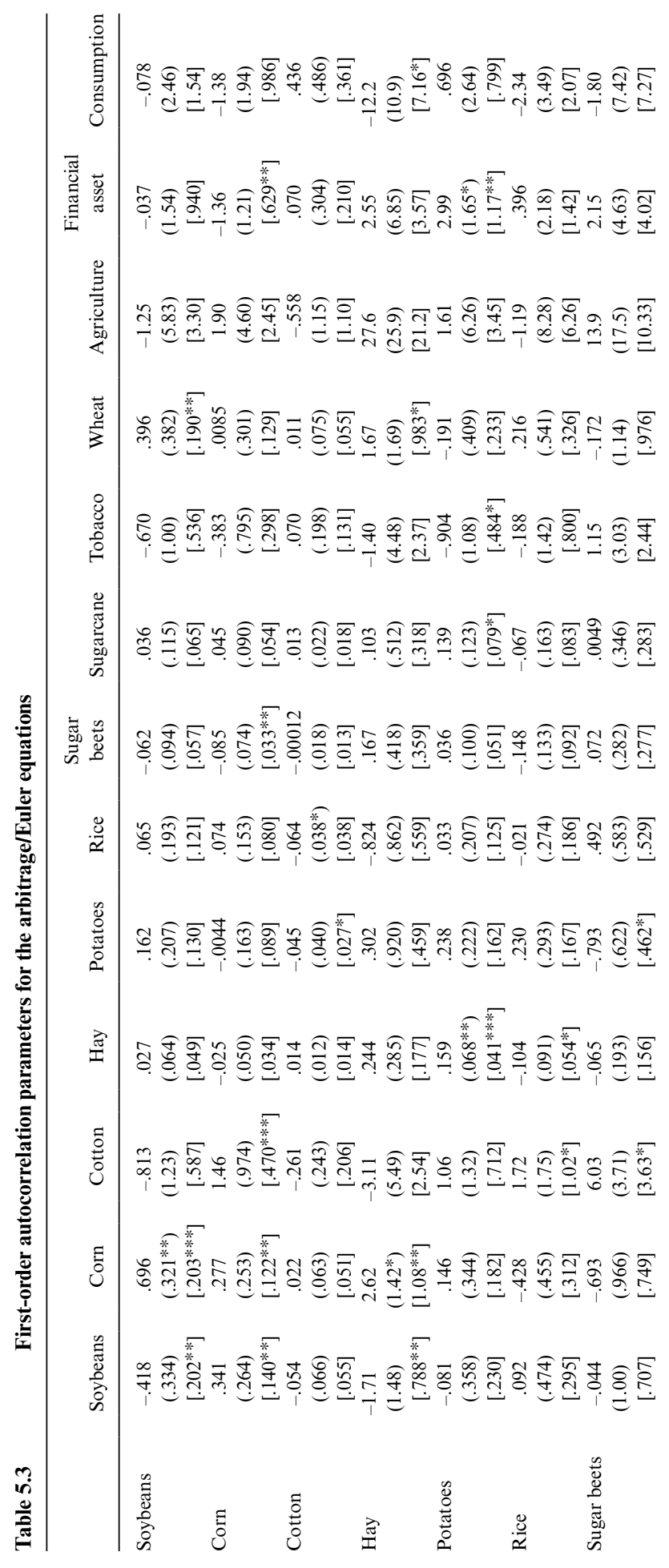




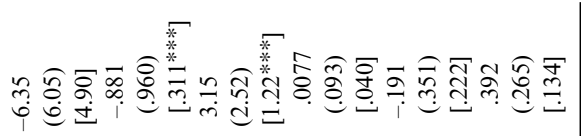

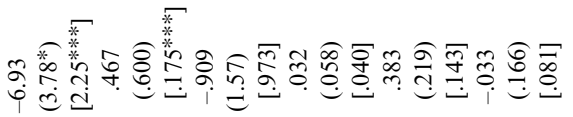

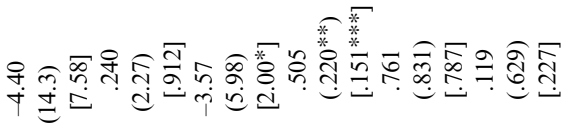

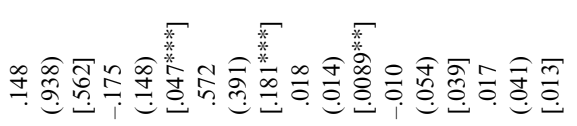

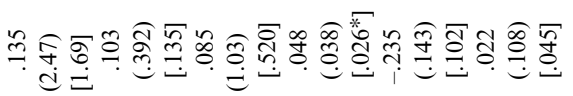

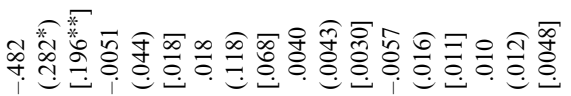

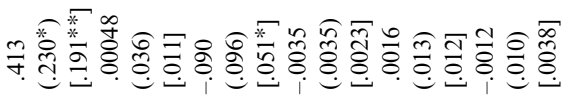

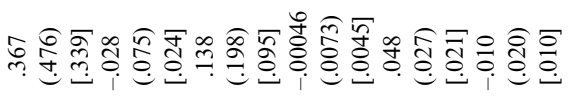

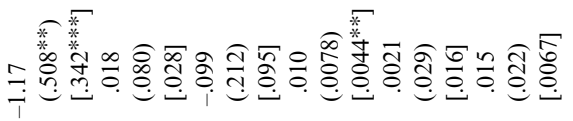

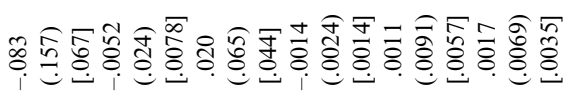

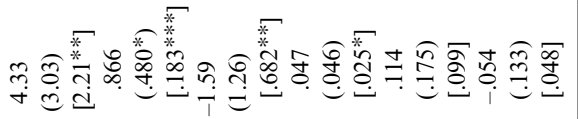

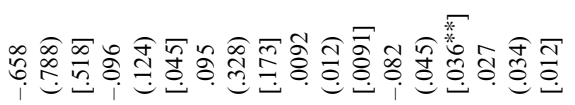

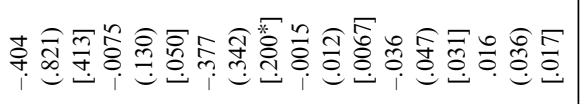

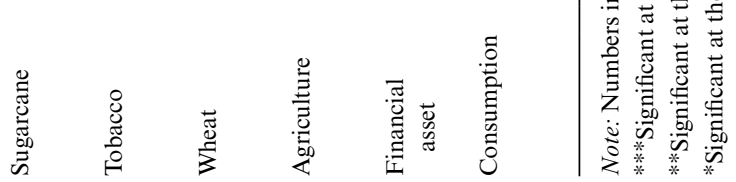




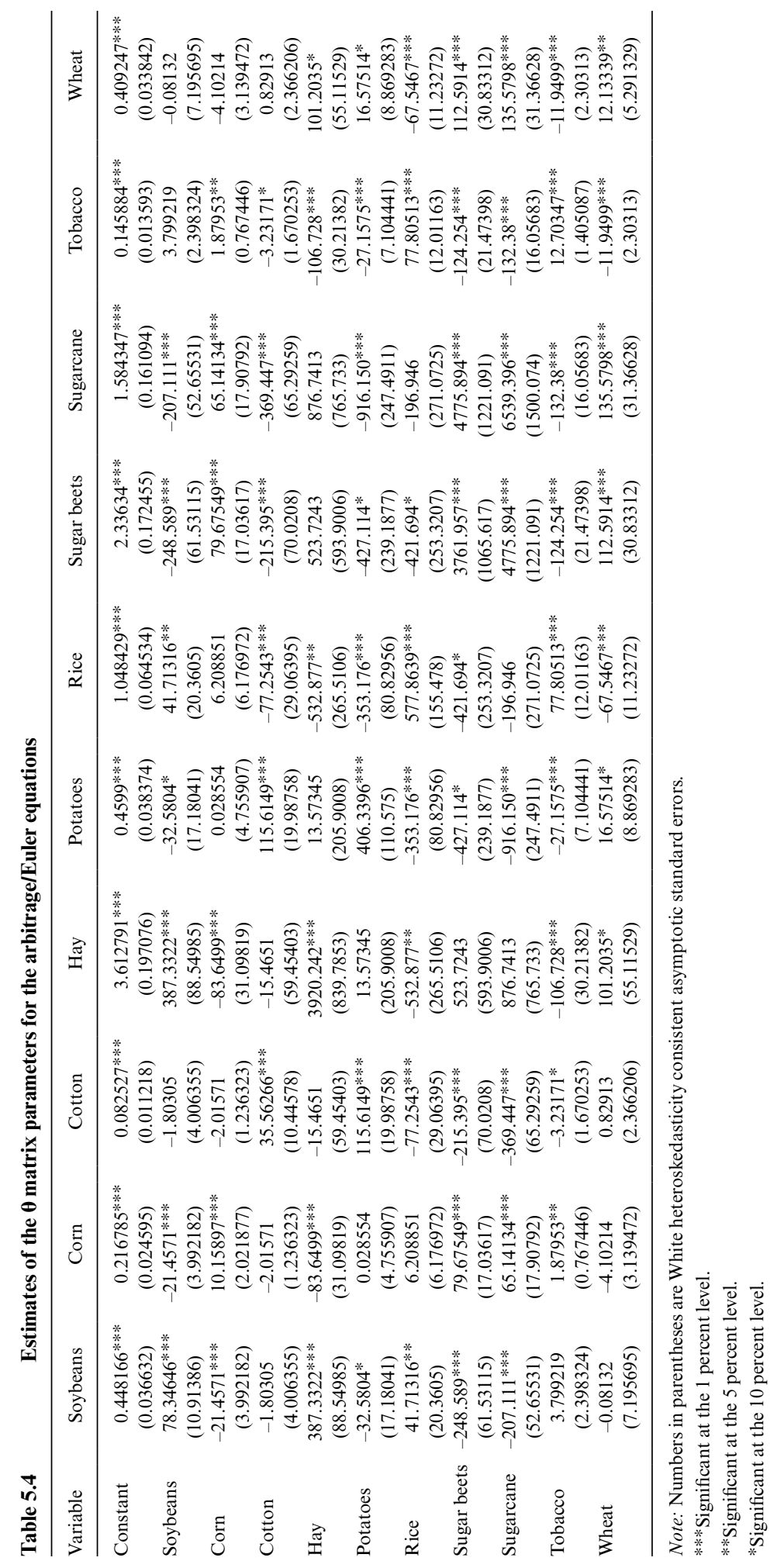


at 0.0 . In other words, the estimated symmetric but not curvature restricted $\boldsymbol{\Theta}$ matrix has four negative Eigen values. As a consequence, the standard errors in table 5.2 are conditional on these inequality restrictions. The estimated structural parameters reported in table 5.4 generate a system of ten linear marginal cost functions that are increasing in planned output levels throughout the sample period. We conclude that this is a coherent and reasonable model of U.S. agricultural production.

The point estimate for the curvature parameter in the quadratic indirect utility function is $\hat{\beta}=6.571 \times 10^{-5}$, with an estimated classical Gaussian asymptotic standard error of $2.793 \times 10^{-7}$ and an estimated White/Huber robust standard error of $5.914 \times 10^{-7}$, both implying a highly significant risk aversion parameter. On the other hand, the point estimates for the quadratic adjustment cost parameters are mixed. The point estimate for adjustment costs on farmland is $\hat{\gamma}_{A}=-4.455 \times 10^{-6}$, with an estimated classical Gaussian asymptotic standard error of $1.985 \times 10^{-6}$ and an estimated White/Huber robust standard error of $1.477 \times 10^{-6}$. In both cases, this is statistically different from zero at the 5 percent significance level, although economically, the sign is not what we would expect a priori. The point estimate for adjustment costs in farm capital is $\hat{\gamma}_{K}=4.012 \times 10^{-11}$, with an estimated classical Gaussian asymptotic standard error of $2.738 \times 10^{-11}$ and an estimated White/Huber robust standard error of $2.383 \times 10^{-11}$. While this has the expected sign, the classical standard error implies this is not statistically different from zero at the 10 percent level of significance, while the robust standard error implies that it marginally is significant at the same level. We suspect that either this level of aggregation across agents cannot capture these effects or else there is at most only a small level of adjustment cost in the farm sector. On the other hand, if there is no adjustment cost mechanism in U.S. agriculture, and if the quadratic indirect utility model is correctly specified, then the Euler equations estimated here are theoretically and empirically correct even with national aggregate data.

\subsection{Conclusions}

This chapter has developed and analyzed a new structural model of variable input use, production, acreage allocations, capital investment, and consumption choices in the U.S. farm sector. The theoretical framework identifies and incorporates the restrictions that are necessary and sufficient to estimate variable input use using only observable data and to aggregate from micro units of behavior to county-, state-, region-, or country-levels of data and analyses. We defined, specified, and estimated a dynamic life-cycle model of decision making under risk. We disciplined the model and associated parameter estimates for risk aversion in agricultural production and investment decisions with the interactions that naturally occur among the available alternative investment and savings opportunities in the economy. 
Current work applies this to state-level data, which should mitigate the issues related to aggregating across different production regions, climates, and output choice sets. We incorporate input and output specific technological change in the empirical model, which should help address issues due to specification errors and structural change that cannot be captured in the aggregate setup. We are specifying and estimating the variable input use decisions and the asset management choices simultaneously to exploit crossequation parameter restrictions and increase the efficiency of our parameter estimates. And last, the data set is in the final stages of being updated to the twenty-first century, which will make the model and empirical analysis more timely and relevant to current farm policies.

One of the central issues guiding agricultural policy is how risk affects choice and welfare. Here, that is manifest in the movement toward general equilibrium found in the cross-moment equations in equation (46) and the cost structure in equation (45). This provides a rich mechanism for policy analysis. The conventional agricultural focus is how policies affect the risk environment and thereby production choice and welfare. Thus, for example, in a partial equilibrium model of the farm sector, one often studies the effects of a particular policy on the risk environment on the portfolio of crop choice (Chavas and Holt 1996). Here, it is clear that the evolution of wealth and income in all forms, and consumption, "cause" production choices. Although this point is not new (e.g., Wright and Hewitt 1994), it has not been formally modeled and estimated.

With the results in table 5.2, one can trace the effects of any policy altering the distribution of agricultural crop income on the choices that restore equilibrium. More specifically, it means that significant responses may be outside of agriculture by changing nonagricultural investment and consumption. These responses likely will alter the normative and positive conclusions of the effects of policies substantially.

Indeed, returning to the example of crop insurance discussed in the introduction, the social value of public insurance will likely be reduced as more margins for adjustment (arbitrage conditions) are included in the analysis. In contrast, an increase in uncertainty (the covariance term) in nonagricultural investments as witnessed recently could increase the demand for risk-reducing agricultural instruments. The key point is that unless one has a model that provides for these interactions, one will not obtain reasonable policy conclusions.

The second general policy insight that can be obtained here is a distinction between long-run and shorter-run effects, which has been one of the foundations of agricultural policy analyses, preceding the seminal work of Nerlove (1958). Yet models in current vogue can only be interpreted as longrun analyses where adjustment costs are zero. This means that one has a natural structural way in the current model to distinguish short-run and long-run elasticities. For example, this implies that policies that raise the 
return to insurance (e.g., through public subsidies) have larger responses in the long run than in the short run.

\section{Appendix A}

Let $\boldsymbol{x} \in \mathscr{X} \subseteq \mathbb{R}_{++}^{n_{x}}$ be an $n_{x}$-vector of variable inputs; let $\boldsymbol{w} \in \mathscr{W} \subseteq \mathbb{R}_{++}^{n_{x}}$ be an $n_{x}$-vector of variable input prices; let $\boldsymbol{y} \in \mathscr{Y} \subseteq \mathbb{R}_{++}^{n_{x}}$ be an $n_{y}$-vector of outputs; let $z \in \mathcal{Z} \subseteq \mathbb{R}_{++}^{n_{x}}$ be an $n_{z}$-vector of quasi-fixed inputs; let $F: \mathscr{X} \times \mathscr{Y} \times \mathcal{Z} \rightarrow \mathbb{R}$ be a transformation function that defines the boundary of a closed, convex production possibilities set with free disposal in inputs and outputs; let $\boldsymbol{X}$ : $\mathscr{W} \times \mathscr{Y} \times \mathcal{Z} \rightarrow \mathscr{X}$, be an $n_{x}$-vector of variable input demand functions; and let $C: \mathscr{W} \times \mathscr{Y} \times \mathcal{Z} \rightarrow \mathbb{R}_{++}$be a variable cost function,

$$
c=C(\boldsymbol{w}, \boldsymbol{y}, \boldsymbol{z}) \equiv \min _{x}\left\{\boldsymbol{w}^{\top} \boldsymbol{x}: F(\boldsymbol{x}, \boldsymbol{y}, \boldsymbol{z}) \leq 0, \boldsymbol{x} \geq 0\right\} \equiv \boldsymbol{w}^{\top} \boldsymbol{X}(\boldsymbol{w}, \boldsymbol{y}, \boldsymbol{z}),
$$

where the symbol ${ }^{\top}$ denotes vector and matrix transposition. The purpose of this appendix is to prove that short-run cost-minimizing variable input demands, $\boldsymbol{x}=\boldsymbol{X}(\boldsymbol{w}, \boldsymbol{y}, \boldsymbol{z})$, can be written in the form $\boldsymbol{x}=\tilde{\boldsymbol{X}}(\boldsymbol{w}, c, \boldsymbol{z})$ if and only if $c=C[\boldsymbol{w}, \boldsymbol{z}, \theta(\boldsymbol{y}, \boldsymbol{z})] \Leftrightarrow F[\boldsymbol{x}, \boldsymbol{z}, \theta(\boldsymbol{y}, \boldsymbol{z})]$.

The neoclassical model of conditional demands for variable inputs with joint production, quasi-fixed inputs, and production uncertainty is

$$
\boldsymbol{X}(\boldsymbol{w}, \boldsymbol{y}, \boldsymbol{z})=\arg \min \left\{\boldsymbol{w}^{\top} \boldsymbol{x}: F(\boldsymbol{x}, \boldsymbol{y}, \boldsymbol{z}) \leq 0, \boldsymbol{x} \geq 0\right\},
$$

where $\boldsymbol{x}$ is an $n_{x}$-vector of positive variable inputs with corresponding positive prices, $\boldsymbol{w} ; \boldsymbol{y}$ is an $n_{y}$-vector of planned outputs; $\boldsymbol{z}$ is an $n_{z}$-vector of quasifixed inputs; $F$ is the real valued transformation function that defines the boundary of a closed, convex production possibilities set with free disposal in the inputs and the outputs; $\boldsymbol{X}$ maps variable input prices, planned outputs, and quasi-fixed inputs into variable input demand functions; and $C(\boldsymbol{w}, \boldsymbol{y}, \boldsymbol{z})$ $\equiv \boldsymbol{w}^{\top} \boldsymbol{X}(\boldsymbol{w}, \boldsymbol{y}, \boldsymbol{z})$, is the positive-valued variable cost function. By Shephard's lemma, we have

$$
\boldsymbol{X}(\boldsymbol{w}, \boldsymbol{y}, \boldsymbol{z})=\nabla_{w} C(\boldsymbol{w}, \boldsymbol{y}, \boldsymbol{z}) \equiv\left(\frac{\partial C}{\partial w_{1}}, \ldots, \frac{\partial C}{\partial w_{n_{x}}}\right)^{\top} .
$$

$\boldsymbol{X}$ is homogeneous of degree zero in $\boldsymbol{w}$ by the derivative property of homogeneous functions. Integrating with respect to $\boldsymbol{w}$ to recover the variable cost function, we obtain

$$
c=C(\boldsymbol{w}, \boldsymbol{y}, \boldsymbol{z}) \equiv \tilde{C}[\boldsymbol{w}, \boldsymbol{y}, \boldsymbol{z}, \theta(\boldsymbol{y}, \boldsymbol{z})],
$$

where $\theta: y \times \mathcal{Z} \rightarrow \mathbb{R}$ is the constant of integration. In the present case, this means that $\theta$ is constant with respect to $\boldsymbol{w}$. In general, $\theta$ is a function of $y$ and $z$, and its structure cannot be identified from the variable input demands 
because it captures that part of the joint production process relating to quasi-fixed inputs and outputs that is separable from the variable inputs.

Under standard conditions, the variable cost function is strictly decreasing in $\boldsymbol{z}$, strictly increasing in $\boldsymbol{y}$, jointly convex in $(\boldsymbol{y}, \boldsymbol{z})$, increasing, concave and homogeneous of degree one in $\boldsymbol{w}$. We are free to choose the sign of $\theta$ so that, with no loss of generality, $\partial \tilde{C} / \partial \theta>0$.

Because $\tilde{C}$ is strictly increasing in $\theta$, a unique inverse exists such that $\theta=\gamma(\boldsymbol{w}, \boldsymbol{y}, \boldsymbol{z}, c)$, where $\gamma: \mathcal{W} \times \mathcal{Y} \times \mathcal{Z} \times \mathbb{R}_{+} \rightarrow \mathbb{R}$, is the inverse of $\tilde{C}$ with respect to $\theta . \gamma(\boldsymbol{w}, \boldsymbol{y}, \boldsymbol{z}, c)$ is called the quasi-indirect production transformation function, analogous to the quasi-indirect utility function of consumer theory (Hausman 1981; Epstein 1982; LaFrance 1985, 1986, 1990, 2004; LaFrance and Hanemann 1989). For all interior and feasible $(\boldsymbol{y}, \boldsymbol{z})$, the function $\gamma$ is strictly increasing in $c$, strictly decreasing and quasi-convex in $\boldsymbol{w}$, and positively homogeneous of degree zero in $(\boldsymbol{w}, c)$.

The following two identities are simple implications of the inverse function theorem:

$$
c \equiv \tilde{C}[\boldsymbol{w}, \boldsymbol{y}, \boldsymbol{z}, \gamma(\boldsymbol{w}, \boldsymbol{y}, \boldsymbol{z}, c)]
$$

and

$$
\theta \equiv \gamma[\boldsymbol{w}, \boldsymbol{y}, \boldsymbol{z}, \tilde{C}(\boldsymbol{w}, \boldsymbol{y}, \boldsymbol{z}, \theta)] .
$$

This lets one write the conditional demands for the variable inputs as

$$
\boldsymbol{x}=\nabla_{w} \tilde{C} \equiv \boldsymbol{G}(\boldsymbol{w}, \boldsymbol{y}, \boldsymbol{z}, c) .
$$

Equation (A7) gives the rationale for writing the factor demands as a function of $c$ as well as $(\boldsymbol{w}, \boldsymbol{y}, \boldsymbol{z})$. Thus, given the preceding regularity conditions for $F$ and $C$, one can always write the system of factor demands as functions of cost.

Now define the quasi-production transformation function by

$$
v(\boldsymbol{w}, \boldsymbol{y}, \boldsymbol{z}) \equiv \min _{\boldsymbol{w} \geq 0}\left\{\gamma\left(\boldsymbol{w}, \boldsymbol{y}, \boldsymbol{z}, \boldsymbol{w}^{\top} \boldsymbol{x}\right\} .\right.
$$

The terminology quasi-production transformation function indicates that $v(\boldsymbol{x}$, $\boldsymbol{y}, \boldsymbol{z})$ only reveals part of the structure of the joint production process. It cannot, and does not, reveal $\theta(\boldsymbol{y}, \boldsymbol{z})$. This is analogous to the situation where one only recovers part of a direct utility function when analyzing the market demands for a subset of consumption goods.

The identity $\theta(\boldsymbol{y}, \boldsymbol{z}) \equiv \boldsymbol{\gamma}\{\boldsymbol{w}, \boldsymbol{y}, \boldsymbol{z}, \tilde{C}[\boldsymbol{w}, \boldsymbol{y}, \boldsymbol{z}, \theta(\boldsymbol{y}, \boldsymbol{z})]\}$ implies

$$
\begin{aligned}
\theta(\boldsymbol{y}, \boldsymbol{z}) & \equiv \gamma\{\boldsymbol{w}, \boldsymbol{y}, \boldsymbol{z}, \tilde{C}[\boldsymbol{w}, \boldsymbol{y}, \boldsymbol{z}, \theta(\boldsymbol{y}, \boldsymbol{z})]\} \geq \min _{\boldsymbol{w} \geq 0}\left\{\gamma\left(\boldsymbol{w}, \boldsymbol{y}, \boldsymbol{z}, \boldsymbol{w}^{\top} \boldsymbol{x}\right)\right\} \\
& \equiv v(\boldsymbol{x}, \boldsymbol{y}, \boldsymbol{z}),
\end{aligned}
$$

for all interior and feasible $(\boldsymbol{x}, \boldsymbol{y}, \boldsymbol{z})$. This inequality follows from the fact that $\theta(\boldsymbol{y}, \boldsymbol{z})$ is feasible but not necessarily optimal in the minimization problem. 
The part of $F(\boldsymbol{x}, \boldsymbol{y}, \boldsymbol{z})$ not contained in $v(\boldsymbol{x}, \boldsymbol{y}, \boldsymbol{z})$ is given by (Diewert 1978; Epstein 1975; Hausman 1981; LaFrance and Hanemmann 1989),

$$
F(\boldsymbol{x}, \boldsymbol{y}, \boldsymbol{z}) \equiv \tilde{F}[\boldsymbol{x}, \boldsymbol{y}, \boldsymbol{z}, \theta(\boldsymbol{y}, \boldsymbol{z})] .
$$

The quasi-production transformation function is the unique solution, $\theta=v(\boldsymbol{x}, \boldsymbol{y}, \boldsymbol{z})$, to the implicit function, $\tilde{F}(\boldsymbol{x}, \boldsymbol{y}, \boldsymbol{z}, \theta)=0$; in other words, $\tilde{F}[\boldsymbol{x}, \boldsymbol{y}, \boldsymbol{z}, \mathrm{v}(\boldsymbol{x}, \boldsymbol{y}, \boldsymbol{z})] \equiv 0$.

The function $v(\boldsymbol{x}, \boldsymbol{y}, \boldsymbol{z})$ in equation (A9) conveys full information about the marginal rates of substitution between variable inputs but only partially so for outputs and quasi-fixed inputs. This is again analogous to the situation in consumption theory when one analyzes only a subset of the goods purchased and consumed. This can be shown by applying the implicit function theorem to $\tilde{F}$, which gives

$$
\begin{aligned}
& \nabla_{x} v(\boldsymbol{x}, \boldsymbol{y}, \boldsymbol{z})=-\frac{\nabla_{x} \tilde{F}[\boldsymbol{x}, \boldsymbol{y}, \boldsymbol{z}, \mathrm{v}(\boldsymbol{x}, \boldsymbol{y}, \boldsymbol{z})]}{\nabla_{\theta} \tilde{F}[\boldsymbol{x}, \boldsymbol{y}, \boldsymbol{z}, \mathrm{v}(\boldsymbol{x}, \boldsymbol{y}, \boldsymbol{z})]}, \\
& \nabla_{y} v(\boldsymbol{x}, \boldsymbol{y}, \boldsymbol{z})=-\frac{\nabla_{y} \tilde{F}[\boldsymbol{x}, \boldsymbol{y}, \boldsymbol{z}, \mathrm{v}(\boldsymbol{x}, \boldsymbol{y}, \boldsymbol{z})]}{\nabla_{\theta} \tilde{F}[\boldsymbol{x}, \boldsymbol{y}, \boldsymbol{z}, \mathrm{v}(\boldsymbol{x}, \boldsymbol{y}, \boldsymbol{z})]}, \\
& \nabla_{z} \mathrm{v}(\boldsymbol{x}, \boldsymbol{y}, \boldsymbol{z})=-\frac{\nabla_{z} \tilde{F}[\boldsymbol{x}, \boldsymbol{y}, \boldsymbol{z}, \mathrm{v}(\boldsymbol{x}, \boldsymbol{y}, \boldsymbol{z})]}{\nabla_{\theta} \tilde{F}[\boldsymbol{x}, \boldsymbol{y}, \boldsymbol{z}, \mathrm{v}(\boldsymbol{x}, \boldsymbol{y}, \boldsymbol{z})]} .
\end{aligned}
$$

This demonstrates that $v$ conveys full information on marginal rates of substitution between variable inputs,

$$
\begin{aligned}
\frac{\partial v(\boldsymbol{x}, \boldsymbol{y}, \boldsymbol{z}) / \partial x_{i}}{\partial v(\boldsymbol{x}, \boldsymbol{y}, \boldsymbol{z}) / \partial x_{j}} & =\frac{\partial \tilde{F}[\boldsymbol{x}, \boldsymbol{y}, \boldsymbol{z}, \mathrm{v}(\boldsymbol{x}, \boldsymbol{y}, \boldsymbol{z})] / \partial x_{i}}{\partial \tilde{F}[\boldsymbol{x}, \boldsymbol{y}, \boldsymbol{z}, \mathrm{v}(\boldsymbol{x}, \boldsymbol{y}, \boldsymbol{z})] / \partial x_{j}} \\
& =\frac{\partial F(\boldsymbol{x}, \boldsymbol{y}, \boldsymbol{z}) / \partial x_{i}}{\partial F(\boldsymbol{x}, \boldsymbol{y}, \boldsymbol{z}) / \partial x_{j}}, \forall i, j=1, \ldots, n_{x},
\end{aligned}
$$

but only partial information on marginal rates of product transformation between outputs,

$$
\begin{aligned}
& \frac{\partial F(\boldsymbol{x}, \boldsymbol{y}, \boldsymbol{z}) / \partial y_{i}}{\partial F(\boldsymbol{x}, \boldsymbol{y}, \boldsymbol{z}) / \partial y_{j}} \\
& \quad=\frac{\partial \tilde{F}[\boldsymbol{x}, \boldsymbol{y}, \boldsymbol{z}, \theta(\boldsymbol{y}, \boldsymbol{z})] / \partial y_{i}+\partial \tilde{F}[\boldsymbol{x}, \boldsymbol{y}, \boldsymbol{z}, \theta(\boldsymbol{y}, \boldsymbol{z})] / \partial \theta \cdot \partial \theta(\boldsymbol{y}, \boldsymbol{z}) / \partial y_{i}}{\partial \tilde{F}[\boldsymbol{x}, \boldsymbol{y}, \boldsymbol{z}, \theta(\boldsymbol{y}, \boldsymbol{z})] / \partial y_{j}+\partial \tilde{F}[\boldsymbol{x}, \boldsymbol{y}, \boldsymbol{z}, \theta(\boldsymbol{y}, \boldsymbol{z})] / \partial \theta \cdot \partial \theta(\boldsymbol{y}, \boldsymbol{z}) / \partial y_{j}} \\
& \quad \neq \frac{\partial v(\boldsymbol{x}, \boldsymbol{y}, \boldsymbol{z}) / \partial y_{i}}{\partial v(\boldsymbol{x}, \boldsymbol{y}, \boldsymbol{z}) / \partial y_{j}}, \forall i, j=1, \ldots, n_{y},
\end{aligned}
$$

and marginal rates of substitution between quasi-fixed inputs, 
(A14)

$$
\begin{aligned}
& \frac{\partial F(\boldsymbol{x}, \boldsymbol{y}, \boldsymbol{z}) / \partial z_{i}}{\partial F(\boldsymbol{x}, \boldsymbol{y}, \boldsymbol{z}) / \partial z_{j}} \\
& \quad=\frac{\partial \tilde{F}[\boldsymbol{x}, \boldsymbol{y}, \boldsymbol{z}, \theta(\boldsymbol{y}, \boldsymbol{z})] / \partial z_{i}+\partial \tilde{F}[\boldsymbol{x}, \boldsymbol{y}, \boldsymbol{z}, \theta(\boldsymbol{y}, \boldsymbol{z})] / \partial \theta \cdot \partial \theta(\boldsymbol{y}, \boldsymbol{z}) / \partial z_{i}}{\partial \tilde{F}[\boldsymbol{x}, \boldsymbol{y}, \boldsymbol{z}, \theta(\boldsymbol{y}, \boldsymbol{z})] / \partial z_{j}+\partial \tilde{F}[\boldsymbol{x}, \boldsymbol{y}, \boldsymbol{z}, \theta(\boldsymbol{y}, \boldsymbol{z})] / \partial \theta \cdot \partial \theta(\boldsymbol{y}, \boldsymbol{z}) / \partial z_{j}} \\
& \quad \neq \frac{\partial v(\boldsymbol{x}, \boldsymbol{y}, \boldsymbol{z}) / \partial z_{i}}{\partial v(\boldsymbol{x}, \boldsymbol{y}, \boldsymbol{z}) / \partial z_{j}}, \forall i, j=1, \ldots, n_{z},
\end{aligned}
$$

This background leads directly to the following result.

Proposition 1. The following functional structures are equivalent:

$$
\begin{aligned}
& x=X(\boldsymbol{w}, \boldsymbol{y}, \boldsymbol{z}) \equiv \tilde{X}(\boldsymbol{w}, c, \boldsymbol{z}) \\
& c=C(\boldsymbol{w}, \boldsymbol{y}, \boldsymbol{z}) \equiv \tilde{C}(\boldsymbol{w}, \boldsymbol{z}, \theta(\boldsymbol{y}, \boldsymbol{z}))
\end{aligned}
$$

and

$$
0=F(\boldsymbol{w}, \boldsymbol{y}, \boldsymbol{z}) \equiv \tilde{F}(\boldsymbol{x}, \boldsymbol{z}, \theta(\boldsymbol{y}, \boldsymbol{z})) .
$$

ProOF. (A16) $\Rightarrow$ (A15). Differentiating equation (A16) with respect to $w$, Shephard's lemma implies,

$$
\boldsymbol{x}=\nabla_{w} \tilde{C} .
$$

$\tilde{C}$ is strictly monotonic in and has a unique inverse with respect to $\theta$, say $\theta=\tilde{\gamma}(\boldsymbol{w}, \boldsymbol{z}, c)$. Substituting this into equation (A18) obtains

$$
\boldsymbol{x}=\nabla_{w} \tilde{C}[\boldsymbol{w}, \boldsymbol{z}, \tilde{\gamma}(\boldsymbol{w}, \boldsymbol{z}, c)] \equiv \tilde{\boldsymbol{X}}(\boldsymbol{w}, c, \boldsymbol{z}) .
$$

$(\mathrm{A} 17) \Rightarrow(\mathrm{A} 15) \Rightarrow(\mathrm{A} 16)$. If the representation of technology has the separable structure in equation (A17), then

(A20) $\arg \min \left\{\boldsymbol{w}^{\top} \boldsymbol{x}: \tilde{F}[\boldsymbol{x}, \boldsymbol{z} \theta(\boldsymbol{y}, \boldsymbol{z})] \leq 0, x \geq 0\right\} \equiv \tilde{\boldsymbol{X}}[\boldsymbol{w}, \boldsymbol{z}, \theta(\boldsymbol{y}, \boldsymbol{z})]$.

This implies that the variable cost function has the separable structure

$$
\boldsymbol{w}^{\top} \tilde{\boldsymbol{X}}[\boldsymbol{w}, \boldsymbol{z}, \theta(\boldsymbol{y}, \boldsymbol{z})] \equiv \tilde{C}[\boldsymbol{w}, \boldsymbol{z}, \theta(\boldsymbol{y}, \boldsymbol{z})] .
$$

$(\mathrm{A} 16) \Rightarrow(\mathrm{A} 17)$. Given equation (A16), the quasi-production transformation function satisfies

$$
\tilde{v}(\boldsymbol{x}, \boldsymbol{z}) \equiv \min _{\mathrm{w} \geq \mathbf{0}}\left\{\tilde{\gamma}\left(\boldsymbol{w}, \boldsymbol{z}, \boldsymbol{w}^{\top} \boldsymbol{x}\right\}\right.
$$

This implies that

$$
\theta(\boldsymbol{y}, \boldsymbol{z}) \equiv \gamma\{\boldsymbol{x}, \boldsymbol{z}, \tilde{C}[\boldsymbol{x}, \boldsymbol{z}, \theta(\boldsymbol{y}, \boldsymbol{z})]\} \geq \tilde{v}(\boldsymbol{x}, \boldsymbol{z}),
$$

for all interior, feasible $(\boldsymbol{x}, \boldsymbol{y}, \boldsymbol{z}) \in \mathscr{X} \times \mathscr{y} \times \mathcal{Z}$, with the boundary of the closed and convex production possibilities set defined by equality on the far right. Because $\tilde{v}$ is independent of $\boldsymbol{y}$, equations (A11) and (A13) imply 
(A24)

$$
\frac{\partial F(\boldsymbol{x}, \boldsymbol{y}, \boldsymbol{z}) / \partial y_{i}}{\partial F(\boldsymbol{x}, \boldsymbol{y}, \boldsymbol{z}) / \partial y_{j}}=\frac{\partial \theta(\boldsymbol{y}, \boldsymbol{z}) / \partial y_{i}}{\partial \theta(\boldsymbol{y}, \boldsymbol{z}) / \partial y_{j}}, \forall i, j=1, \ldots, n_{y} .
$$

Hence, the marginal rates of transformation between outputs are independent of variable inputs,

$$
\begin{gathered}
\frac{\partial}{\partial x_{k}}\left[\frac{\partial F(\boldsymbol{x}, \boldsymbol{y}, \boldsymbol{z}) / \partial y_{i}}{\partial F(\boldsymbol{x}, \boldsymbol{y}, \boldsymbol{z}) / \partial y_{j}}\right]=\frac{\partial}{\partial x_{k}}\left[\frac{\partial \theta(\boldsymbol{y}, \boldsymbol{z}) / \partial y_{i}}{\partial \theta(\boldsymbol{y}, \boldsymbol{z}) / \partial y_{j}}\right]=0, \\
\forall i, j=1, \ldots, n_{y}, \forall k=1, \ldots, n_{x},
\end{gathered}
$$

Thus, $\boldsymbol{y}$ is separable from $\boldsymbol{x}$ in the joint production transformation function (Goldman and Uzawa 1964, Lemma 1); that is, $F(\boldsymbol{x}, \boldsymbol{y}, \boldsymbol{z})=\tilde{F}[\boldsymbol{x}, \boldsymbol{z}, \theta(\boldsymbol{y}, \boldsymbol{z})]$.

\section{Appendix B}

\section{Sufficiency Algebra for Proposition 2}

Define the function $\omega: \mathbb{R}_{+} \times \mathbb{R} \rightarrow \mathbb{R}$ by

$$
\omega(x, y)=y+\int_{0}^{x}\left[\lambda(s)+\omega(s, y)^{2}\right] d s,
$$

where $\lambda: \mathbb{R} \rightarrow \mathbb{R}$ is an arbitrary smooth function, and $w$ is subject to the pair of initial conditions, $w(0, y)=y$ and $\partial w(0, y) / \partial x=\lambda(0)+y^{2}$, to ensure that the definition is unique and smooth. Given two arbitrary smooth functions $\eta: \mathbb{R}_{++}^{n_{x}} \rightarrow \mathbb{R}_{+}$and $\theta: \mathbb{R}^{n_{z}} \times \mathbb{R}^{n_{y}} \rightarrow \mathbb{R}$, by the Leibniz rule of differentiation, we have

$$
\frac{\partial \omega[\eta(\boldsymbol{w}), \theta(\boldsymbol{z}, \overline{\boldsymbol{y}})]}{\partial \boldsymbol{w}}=\left\{\lambda[\eta(\boldsymbol{w})]+\omega[\eta(\boldsymbol{w}), \theta(\boldsymbol{z}, \overline{\boldsymbol{y}})]^{2}\right\} \frac{\partial \eta(w)}{\partial w} .
$$

Given a monotonic, smooth function $f: \mathbb{R}_{++} \rightarrow \mathbb{C}, f^{\prime} \neq 0$, define the relationship between $f$ and $\omega$ by $f=(\alpha \omega+\beta) /(\gamma \omega+\delta), \alpha, \beta, \gamma, \delta: \mathbb{R}_{+_{+}}^{n_{*}} \rightarrow \mathbb{C}$, and $\alpha \delta-\beta \gamma \equiv 1$. Let the cost function be $c: \mathbb{R}_{++}^{n_{x}} \times \mathbb{R}_{++}^{n_{z}} \times \mathbb{R}_{++}^{n_{y}} \rightarrow \mathbb{R}_{++}$ and denote an arbitrary positive-valued, $1^{\circ}$ homogeneous, increasing, and concave deflator by $\pi: \mathbb{R}_{++}^{n_{x}} \rightarrow \mathbb{R}_{++}$. The projective transformation group representation of any exactly aggregable ex ante cost function is

$$
f\left[\frac{c(w, z, \bar{y})}{\pi(w)}\right]=\frac{\alpha(w) \omega[\eta(w), \theta(z, \bar{y})]+\beta(w)}{\gamma(w) \omega[\eta(w), \theta(z, \bar{y})]+\delta(w)} .
$$

Hereafter, suppress all arguments of all functions and use bold italics subscripts to denote vector-valued partial derivatives. For example, rewrite equation (B2) compactly as $\omega_{w}=\left(\lambda+\omega^{2}\right) \eta_{w}$.

The inverse of equation (B3) with respect to $\omega$ is $\omega=(\delta f-\beta) /(-\gamma f+\alpha)$. Combine this with the identification normalization $\alpha \delta-\beta \gamma \equiv 1$ to obtain the following: 
(B4) $\gamma \omega+\delta=\gamma\left(\frac{\delta f-\beta}{-\gamma f+\alpha}\right)+\delta=\frac{\gamma \delta f-\beta \gamma-\gamma \delta f+\alpha \delta}{-\gamma f+\alpha}=\frac{1}{-\gamma f+\alpha}$,

or equivalently, $-\gamma f+\alpha=1 /(\gamma \omega+\delta)$. Multiply each side of this by the corresponding side of equation (B3) to obtain $(-\gamma f+\alpha) f=(\alpha \omega+\beta) /(\gamma \omega+\delta)^{2}$. These relationships are used in what follows to simplify expressions.

Our task is to differentiate equation (B3) with respect to $w$, combine terms, and rewrite the expression that results so that the elements of $\left\{1, f, f^{2}\right\}$ appear on the right. Differentiating gives

$$
\begin{aligned}
f^{\prime} \cdot\left(\frac{c_{w}}{\pi}-\frac{c \pi_{w}}{\pi^{2}}\right)= & \frac{\left(\alpha_{w} \omega+\alpha \omega_{w}+\beta_{w}\right)}{(\gamma w+\delta)}-\frac{(\alpha \omega+\beta)\left(\gamma_{w} \omega+\gamma \omega_{w}+\delta_{w}\right)}{(\gamma w+\delta)^{2}} \\
= & (-\gamma f+\alpha)\left[\alpha_{w} \omega+\alpha\left(\lambda+\omega^{2}\right) \eta_{w}+\beta_{w}\right] \\
& -(-\gamma f+\alpha) f\left[\gamma_{w} \omega+\gamma\left(\lambda+\omega^{2}\right) \eta_{w}+\delta_{w}\right] .
\end{aligned}
$$

The second line follows from $1 /(\gamma \omega+\delta)=-\gamma f+\alpha,(\alpha \omega+\beta) /(\gamma \omega+\delta)^{2}=$ $(-\gamma f+\alpha) f$, and $\omega_{w}=\left(\lambda+\omega^{2}\right) \eta_{w}$. Group terms in $\omega$ on the second line of equation (B5) to obtain

$$
\begin{aligned}
f^{\prime} \cdot\left(\frac{c_{w}}{\pi}-\frac{c \pi_{w}}{\pi^{2}}\right)= & (-\gamma f+\alpha)\left[\beta_{w}+\alpha \lambda \eta_{w}-\left(\delta_{w}+\gamma \lambda \eta_{w}\right) f\right] \\
& +(-\gamma f+\alpha)\left(\alpha_{w}-\gamma_{w} f\right) \omega+(-\gamma f+\alpha)^{2} \eta_{w} \omega^{2}
\end{aligned}
$$

Substituting $\omega=(\delta f-\beta) /(-\gamma f+\alpha)$ into the second line of equation (B6) now leads to

$$
\begin{aligned}
f^{\prime} \cdot\left(\frac{c_{w}}{\pi}-\frac{c \pi_{w}}{\pi^{2}}\right)= & (-\gamma f+\alpha)\left[\beta_{w}+\alpha \lambda \eta_{w}-\left(\delta_{w}+\gamma \lambda \eta_{w}\right) f\right] \\
& +(-\gamma f+\alpha)\left(\alpha_{w}-\gamma_{w} f\right)\left(\frac{\delta f-\beta}{-\gamma f+\alpha}\right) \\
& +(-\gamma f+\alpha)^{2} \eta_{w}\left(\frac{\delta f-\beta}{-\gamma f+\alpha}\right)^{2} \\
= & (-\gamma f+\alpha)\left[\beta_{w}+\alpha \lambda \eta_{w}-\left(\delta_{w}+\gamma \lambda \eta_{w}\right) f\right] \\
& +\left(\alpha_{w}-\gamma_{w} f\right)(\delta f-\beta)+\eta_{w}(\delta f-\beta)^{2} .
\end{aligned}
$$

Expanding the quadratic forms and grouping terms in $f$ in the last line of equation (B7) gives 


$$
\text { (B8) } \begin{aligned}
f^{\prime} \cdot\left(\frac{c_{w}}{\pi}-\frac{c \pi_{w}}{\pi^{2}}\right)= & -\gamma f\left[\beta_{w}+\alpha \lambda \eta_{w}-\left(\delta_{w}+\gamma \lambda \eta_{w}\right) f\right] \\
+\alpha\left[\beta_{w}+\alpha \lambda \eta_{w}-\left(\delta_{w}+\gamma \lambda \eta_{w}\right) f\right] & -\alpha_{w} \beta+\left(\alpha_{w} \delta+\gamma_{w} \beta\right) f-\gamma_{w} \delta f^{2} \\
& +\eta_{w}\left(\beta^{2}-2 \beta \delta f+\delta^{2} f^{2}\right) \\
= & \alpha\left(\beta_{w}+\alpha \lambda \eta_{w}\right)-\left(\gamma \beta_{w}+\alpha \delta_{w}+2 \alpha \gamma \lambda \eta_{w}\right) f \\
& +\gamma\left(\delta_{w}+\gamma \lambda \eta_{w}\right) f^{2}-\alpha_{w} \beta+\eta_{w} \beta^{2} \\
& +\left(\alpha_{w} \delta+\gamma w \beta-2 \beta \delta \eta_{w}\right) f+\left(-\gamma_{w} \delta+\eta_{w} \delta^{2}\right) f^{2} \\
= & \alpha \beta_{w}-\beta \alpha_{w}+\left(\alpha^{2} \lambda+\beta^{2}\right) \eta_{w} \\
& -\left[\alpha \delta_{w}-\delta \alpha_{w}+\gamma \beta_{w}-\beta \gamma_{w}+2(\alpha \gamma \lambda+\beta \delta) \eta_{w}\right] f \\
& +\left[\gamma \delta_{w}-\delta \gamma_{w}+\left(\gamma^{2} \lambda+\delta^{2}\right) \eta_{w}\right] f^{2} .
\end{aligned}
$$

Grouping terms in $\eta_{w}$ as well gives

$$
\begin{aligned}
f^{\prime} \cdot\left(\frac{c_{w}}{\pi}-\frac{c \pi_{w}}{\pi^{2}}\right)= & \alpha \beta_{w}-\beta \alpha_{w}-\left(\alpha \delta_{w}-\delta \alpha_{w}+\gamma \beta_{w}-\beta \gamma_{w}\right) f \\
& +\left(\gamma \delta_{w}-\delta \gamma_{w}\right) f^{2}+\left[(\delta f-\beta)^{2}+\lambda(-\gamma f+\alpha)^{2}\right] \eta_{w}
\end{aligned}
$$

Finally, solving for $c_{w}=\boldsymbol{x}$ gives

$$
\begin{aligned}
& \boldsymbol{x}=\frac{\pi_{w}}{\pi} c+\pi\left\{\left[\alpha \beta_{w}-\beta \alpha_{w}+\left(\alpha^{2} \lambda+\beta^{2}\right) \eta_{w}\right] \frac{1}{f^{\prime}}\right. \\
&-\left[\alpha \delta_{w}-\delta \alpha_{w}+\gamma \beta_{w}-\beta \gamma_{w}+2(\alpha \gamma \lambda+\beta \delta) \eta_{w}\right] \frac{f}{f^{\prime}} \\
&\left.+\left[\gamma \delta_{w}-\delta \gamma_{w}+\left(\gamma^{2} \lambda+\delta^{2}\right) \eta_{w}\right] \frac{f^{2}}{f^{\prime}}\right\} \\
&=\frac{\pi_{w}}{\pi} c+\frac{\pi}{f^{\prime}}\left\{\alpha \beta_{w}-\beta \alpha_{w}-\left(\alpha \delta_{w}-\delta \alpha_{w}+\gamma \beta_{w}-\beta \gamma_{w}\right) f\right. \\
&\left.+\left(\gamma \delta_{w}-\delta \gamma_{w}\right) f^{2}+\left[(\delta f-\beta)^{2}+(-\gamma f+\alpha)^{2} \lambda\right] \eta_{w}\right\} .
\end{aligned}
$$

\section{Appendix C}

\section{Specifying the Cost Function}

The first $n_{x}-1$ variable input prices, $\boldsymbol{w}$, and total variable cost, $c$, are normalized by the average wage rate for hired farm labor, $w_{n_{x}}$. We consider the following transformation of normalized variable cost, which nests the price 
independent generalized logarithmic (PIGLOG) and price independent generalized linear (PIGL) class of models,

$$
f(c)=\frac{\left(c^{\kappa}+\kappa-1\right)}{\kappa}, f^{\prime}(c)=c^{\kappa}, f^{\prime \prime}(c)=(\kappa-1) c^{\kappa-2}, \kappa \in \mathbb{R}_{+} .
$$

This includes all of the real-valued Gorman functional forms, with $f(c)=c$ when $\kappa=1$, and $\lim _{\kappa \rightarrow 0} f(c)=1+\ln c$. Therefore, the highest rank that the variable input demands can achieve is three (Gorman 1981; Lewbel 1987; LaFrance and Pope 2009).

Previous empirical work considered translated Box-Cox functions of input prices, $\left(w_{i}^{\lambda}+\lambda-1\right) / \lambda, \lambda \in[0,1], i=1, \ldots, n_{x}-1$, to nest models with that have log prices, power functions of prices, and are linear prices. In the national model $\lambda=1$ is optimal on this interval and for our data set. Hence, we restrict attention here to normalized input prices. Our previous empirical results using this data at state- and national-levels of aggregation and various levels of aggregation across inputs, suggests quite strongly that rank three overparameterizes this data set (Ball et al. 2010). Hence, we focus here on rank two:

$$
\begin{aligned}
f[c(\boldsymbol{w}, A, K, \boldsymbol{a}, \overline{\boldsymbol{Y}})] & =\alpha(\boldsymbol{w}, A, K)+\beta(\boldsymbol{w}) \theta(A, K, \boldsymbol{a}, \overline{\boldsymbol{Y}}), \\
\Leftrightarrow \tilde{\theta}(\boldsymbol{w}, c, A, K) & =\frac{f(c)-\alpha(\boldsymbol{w}, A, K)}{\beta(\boldsymbol{w})}, \\
\alpha(\boldsymbol{w}, A, K) & =\left(\alpha_{10}+\boldsymbol{\alpha}_{1}^{\top} \boldsymbol{w}\right) A+\left(\alpha_{20}+\boldsymbol{\alpha}_{2}^{\top} \boldsymbol{w}\right) K, \\
\beta(\boldsymbol{w}) & =\sqrt{\boldsymbol{w}^{\top} \boldsymbol{B} \boldsymbol{w}+2 \boldsymbol{\gamma}^{\top} \boldsymbol{w}+1}, \\
\overline{\boldsymbol{Y}} & =\overline{\boldsymbol{y}}^{\bullet} \boldsymbol{a}_{t}=\left[\bar{y}_{1} a_{1} \ldots \bar{y}_{n_{y}} a_{n_{y}}\right]^{\top},
\end{aligned}
$$

where $\bar{y}_{i}$ is the expected (planned) yield for the $i^{t h}$ crop, $a_{i}$ is the acreage planted to this crop, and the symbol $\bullet$ denotes the Hadamard/Schur product for matrices and vectors. This appendix identifies restrictions on the parameters in equation $(\mathrm{Cl})$ that are necessary and sufficient for economic regularity of the variable cost function.

Monotonicity in $w$ :

$$
\begin{aligned}
c^{\kappa-1} \frac{\partial c}{\partial \boldsymbol{w}} & =\boldsymbol{\alpha}_{1} A+\boldsymbol{\alpha}_{2} K+\frac{\theta}{\beta}(\boldsymbol{B} \boldsymbol{w}+\boldsymbol{\gamma}) \geq 0 \\
\Leftrightarrow \tilde{\boldsymbol{x}} & =c^{1-\kappa}\left[\boldsymbol{\alpha}_{1} A+\boldsymbol{\alpha}_{2} K+\left(\frac{f-\alpha}{\beta^{2}}\right)(\boldsymbol{B} \boldsymbol{w}+\boldsymbol{\gamma})\right] \geq \mathbf{0},
\end{aligned}
$$

where $\tilde{\boldsymbol{x}}=\left[x_{1} \ldots x_{n_{x^{-}}-1}\right]^{\top}$ is the $\left(n_{x}-1\right)$-vector of the first $n_{x}-1$ input quantities, excluding labor. 
Concavity in $\boldsymbol{w}$ :

$$
\begin{aligned}
& (\kappa-1) c^{\kappa-2} \frac{\partial c}{\partial \boldsymbol{w}} \frac{\partial c}{\partial \boldsymbol{w}^{\top}}+c^{\kappa-1} \frac{\partial^{2} c}{\partial \boldsymbol{w} \partial \boldsymbol{w}^{\top}}=\frac{\theta}{\beta} \boldsymbol{B}-\frac{\theta}{\beta^{3}}(\boldsymbol{B} \boldsymbol{w}+\boldsymbol{\gamma})(\boldsymbol{B} \boldsymbol{w}+\boldsymbol{\gamma})^{\top} \\
& \Leftrightarrow \frac{\partial^{2} c}{\partial \boldsymbol{w} \partial \boldsymbol{w}^{\top}}=\left(\frac{1-\kappa}{c}\right) \tilde{\boldsymbol{x}} \tilde{\boldsymbol{x}}^{\top}+c^{1-\kappa}\left(\frac{f-\alpha}{\beta^{2}}\right)\left[\boldsymbol{B}-\frac{(\boldsymbol{B} \boldsymbol{w}+\boldsymbol{\gamma})(\boldsymbol{B} \boldsymbol{w}+\boldsymbol{\gamma})^{\top}}{\beta^{2}}\right],
\end{aligned}
$$

The first matrix on the right-hand side of the second line is rank one and is negative semidefinite if and only if $\kappa \geq 1$. The matrix in square brackets on the far right of the second line will be positive semidefinite if $\boldsymbol{B}=\boldsymbol{L} \boldsymbol{L}^{\top}+\boldsymbol{\gamma} \boldsymbol{\gamma}^{\top}$, where $\boldsymbol{L}$ is a triangular matrix with nonzero main diagonal elements. This makes the following $n_{x} \times n_{x}$ matrix positive definite:

$$
\left[\begin{array}{ll}
\boldsymbol{B} & \boldsymbol{\gamma} \\
\boldsymbol{\gamma}^{\top} & 1
\end{array}\right]=\left[\begin{array}{ll}
\boldsymbol{L} & \boldsymbol{\gamma} \\
0^{\top} & 1
\end{array}\right]\left[\begin{array}{ll}
\boldsymbol{L}^{\top} & 0^{\top} \\
\boldsymbol{\gamma}^{\top} & 1
\end{array}\right]=\left[\begin{array}{ll}
\boldsymbol{L} \boldsymbol{L}^{\top}+\boldsymbol{\gamma} \boldsymbol{\gamma}^{\top} & \boldsymbol{\gamma} \\
\boldsymbol{\gamma}^{\top} & 1
\end{array}\right],
$$

since it gives a Choleski factorization of the matrix on the left. It follows from this that

$$
\boldsymbol{w}^{\top} \boldsymbol{B} \boldsymbol{W}+2 \boldsymbol{\gamma}^{\top} \boldsymbol{w}+1=\left[\begin{array}{ll}
\boldsymbol{w}^{\top} & 1
\end{array}\right]\left[\begin{array}{cc}
\boldsymbol{B} & \boldsymbol{\gamma} \\
\boldsymbol{\gamma}^{\top} & 1
\end{array}\right]\left[\begin{array}{c}
\boldsymbol{w} \\
1
\end{array}\right]>0 \forall \boldsymbol{w} \in \mathbb{R}_{++}^{n_{x}-1},
$$

and

$$
\left[\boldsymbol{B}-\frac{(\boldsymbol{B} \boldsymbol{w}+\boldsymbol{\gamma})(\boldsymbol{B} \boldsymbol{w}+\boldsymbol{\gamma})^{\top}}{\boldsymbol{w}^{\top} \boldsymbol{B} \boldsymbol{w}+2 \boldsymbol{\gamma}^{\top} \boldsymbol{w}+1}\right]
$$

is positive semidefinite, by the Cauchy-Schwartz inequality in $n_{x}$-dimensional Euclidean space. Given this, the second term on the right-hand side of the second line of equation (12) will be negative semidefinite if and only if $f \leq \alpha$.

Constant returns to scale $(\mathrm{CRS})$ :

$$
\theta \equiv \frac{\partial \theta}{\partial A} A+\frac{\partial \theta}{\partial K} K+\frac{\partial \theta}{\partial \boldsymbol{a}^{\top}} \boldsymbol{a}+\frac{\partial \theta}{\partial \overline{\boldsymbol{Y}}^{\top}} \overline{\boldsymbol{Y}}
$$

We believe that we have a much more accurate measure of capital than we do of land. Hence, we normalize $\theta$ by the value of capital rather than land in farms.

Monotonicity in $(A, K, \boldsymbol{a}, \overline{\boldsymbol{Y}})$ :

$$
\begin{aligned}
& c^{\kappa-1} \frac{\partial c}{\partial A}=\alpha_{10}+\boldsymbol{\alpha}_{1}^{\top} w+\beta \frac{\partial \theta}{\partial A} \leq 0, c^{\kappa-1} \frac{\partial c}{\partial K}=\alpha_{20}+\boldsymbol{\alpha}_{2}^{\top} w+\beta \frac{\partial \theta}{\partial K} \leq 0, \\
& c^{\kappa-1} \frac{\partial c}{\partial \boldsymbol{a}}=\beta \frac{\partial \theta}{\partial \boldsymbol{a}} \leq 0, c^{\kappa-1} \frac{\partial c}{\partial \overline{\boldsymbol{Y}}}=\beta \frac{\partial \theta}{\partial \overline{\boldsymbol{Y}}} \geq \mathbf{0} .
\end{aligned}
$$


Joint Convexity in $(A, K, a, \bar{Y})$ :

$$
\begin{gathered}
{\left[\begin{array}{cccc}
\frac{\partial^{2} c}{\partial A^{2}} & \frac{\partial^{2} c}{\partial A \partial K} & \frac{\partial^{2} c}{\partial A \partial \boldsymbol{a}^{\top}} & \frac{\partial^{2} c}{\partial A \partial \overline{\boldsymbol{Y}}^{\top}} \\
\frac{\partial^{2} c}{\partial K \partial A} & \frac{\partial^{2} c}{\partial K^{2}} & \frac{\partial^{2} c}{\partial K \partial \boldsymbol{a}} & \frac{\partial^{2} c}{\partial K \partial \overline{\boldsymbol{Y}}^{\top}} \\
\frac{\partial^{2} c}{\partial \boldsymbol{a} \partial A} & \frac{\partial^{2} c}{\partial \boldsymbol{a} \partial K} & \frac{\partial^{2} c}{\partial \boldsymbol{a} \partial \boldsymbol{a}^{\top}} & \frac{\partial^{2} c}{\partial \boldsymbol{a} \partial \overline{\boldsymbol{Y}}^{\top}} \\
\frac{\partial^{2} c}{\partial \overline{\boldsymbol{Y}} \partial A} & \frac{\partial^{2} c}{\partial K \partial \overline{\boldsymbol{Y}}} & \frac{\partial^{2} c}{\partial \boldsymbol{a} \partial \overline{\boldsymbol{Y}}} & \frac{\partial^{2} c}{\partial \overline{\boldsymbol{Y}} \partial \overline{\boldsymbol{Y}}^{\top}}
\end{array}\right]} \\
=\frac{1-\kappa}{c}\left[\begin{array}{l}
\frac{\partial c}{\partial A} \\
\frac{\partial c}{\partial K} \\
\frac{\partial c}{\partial \boldsymbol{a}} \\
\frac{\partial c}{\partial \overline{\boldsymbol{Y}}}
\end{array}\right]\left[\begin{array}{l}
\frac{\partial c}{\partial A} \\
\frac{\partial c}{\partial K} \\
\frac{\partial c}{\partial \boldsymbol{a}} \\
\frac{\partial c}{\partial \overline{\boldsymbol{Y}}}
\end{array}\right]^{\top}\left[\begin{array}{llll}
\frac{\partial^{2} \theta}{\partial A^{2}} & \frac{\partial^{2} \theta}{\partial A \partial K} & \frac{\partial^{2} \theta}{\partial A \partial \boldsymbol{a}^{\top}} & \frac{\partial^{2} \theta}{\partial A \partial \overline{\boldsymbol{Y}}^{\top}} \\
\frac{\partial^{2} \theta}{\partial K \partial A} & \frac{\partial^{2} \theta}{\partial K^{2}} & \frac{\partial^{2} \theta}{\partial K \partial \boldsymbol{a}^{\top}} & \frac{\partial^{2} \theta}{\partial K \partial \overline{\boldsymbol{Y}}^{\top}} \\
\frac{\partial^{2} \theta}{\partial \boldsymbol{a} \partial A} & \frac{\partial^{2} \theta}{\partial \boldsymbol{a} \partial K} & \frac{\partial^{2} \theta}{\partial \boldsymbol{a} \partial \boldsymbol{a}^{\top}} & \frac{\partial^{2} \theta}{\partial \boldsymbol{a} \partial \overline{\boldsymbol{Y}}^{\top}} \\
\frac{\partial^{2} \theta}{\partial \overline{\boldsymbol{Y}} \partial A} & \frac{\partial^{2} \theta}{\partial K \partial \overline{\boldsymbol{Y}}} & \frac{\partial^{2} \theta}{\partial \boldsymbol{a} \partial \overline{\boldsymbol{Y}}} & \frac{\partial^{2} \theta}{\partial \overline{\boldsymbol{Y}} \partial \overline{\boldsymbol{Y}}^{\top}}
\end{array}\right]
\end{gathered}
$$

The first matrix on the right is rank one and will be positive semidefinite if and only if $\kappa \leq 1$. Therefore, $c(\boldsymbol{w}, A, K, \boldsymbol{a}, \overline{\boldsymbol{Y}})$ will be concave in $\boldsymbol{w}$ and jointly convex in $(A, K, \boldsymbol{a}, \overline{\boldsymbol{Y}})$ more than locally if and only if $\kappa=1$. We estimated the rank two model using the Box-Cox transformation on cost. The NL3SLS/GMM point estimate for $\kappa$ is 1.124 with a classical (Gaussian) asymptotic standard error of .152 and a White/Huber heteroskedasticity consistent standard error of .111. We cannot reject a null hypothesis of $\kappa=1$ in either case at the 25 percent significance level. Hence, in this chapter, we restrict our attention to $\kappa=1$.

Given this restriction, the cost function will be jointly convex in $(A, K, \boldsymbol{a}, \overline{\boldsymbol{Y}})$ if and only if the Hessian matrix for $\theta$,

$$
\left[\begin{array}{llll}
\frac{\partial^{2} \theta}{\partial A^{2}} & \frac{\partial^{2} \theta}{\partial A \partial K} & \frac{\partial^{2} \theta}{\partial A \partial \boldsymbol{a}^{\top}} & \frac{\partial^{2} \theta}{\partial A \partial \overline{\boldsymbol{Y}}^{\top}} \\
\frac{\partial^{2} \theta}{\partial K \partial A} & \frac{\partial^{2} \theta}{\partial K^{2}} & \frac{\partial^{2} \theta}{\partial K \partial \boldsymbol{a}^{\top}} & \frac{\partial^{2} \theta}{\partial K \partial \overline{\boldsymbol{Y}}^{\top}} \\
\frac{\partial^{2} \theta}{\partial \boldsymbol{a} \partial A} & \frac{\partial^{2} \theta}{\partial \boldsymbol{a} \partial K} & \frac{\partial^{2} \theta}{\partial \boldsymbol{a} \partial \boldsymbol{a}^{\top}} & \frac{\partial^{2} \theta}{\partial \boldsymbol{a} \partial \overline{\boldsymbol{Y}}^{\top}} \\
\frac{\partial^{2} \theta}{\partial \overline{\boldsymbol{Y}} \partial A} & \frac{\partial^{2} \theta}{\partial K \partial \overline{\boldsymbol{Y}}} & \frac{\partial^{2} \theta}{\partial \overline{\boldsymbol{Y}} \partial \boldsymbol{a}^{\top}} & \frac{\partial^{2} \theta}{\partial \overline{\boldsymbol{Y}} \partial \overline{\boldsymbol{Y}}^{\top}}
\end{array}\right] .
$$


is positive semidefinite. Given these considerations, the specification for $\theta$ employed in the chapter is

(C9) $\theta\left(A_{t}, K_{t}, \boldsymbol{a}_{t}, \overline{\boldsymbol{Y}}_{t}\right)=$

$$
-\theta_{1} A_{t}-\theta_{2} K_{t}-\boldsymbol{\theta}_{3}^{\top} \boldsymbol{a}_{t}+\boldsymbol{\theta}_{4}^{\top} \overline{\boldsymbol{Y}}_{t}+\frac{1}{2}\left(\frac{\theta_{5} A_{t}^{2}+\boldsymbol{a}_{t}^{\top} \boldsymbol{\Theta}_{6} \boldsymbol{a}_{t}+\overline{\boldsymbol{Y}}_{t}^{\top} \boldsymbol{\Theta}_{7} \overline{\boldsymbol{Y}}_{t}}{K_{t}}\right),
$$

where $\theta_{1}, \theta_{2}, \theta_{5}>0, \boldsymbol{\theta}_{3}, \boldsymbol{\theta}_{4}>\mathbf{0}$, and $\boldsymbol{\Theta}_{6}, \boldsymbol{\Theta}_{7}$ are symmetric and positive semidefinite. The implied constraints for monotonicity can be written as

$$
\begin{aligned}
& \frac{\partial c_{t}}{\partial A_{t}}<\mathbf{0} \forall t \Leftrightarrow \min _{t}\left(\theta_{1}-\theta_{5} \frac{A_{t}}{K_{t}}\right)>\max _{t}\left(\frac{\alpha_{0}+\boldsymbol{\alpha}_{1}^{\top} \boldsymbol{w}_{t}}{\sqrt{\beta(\boldsymbol{w})}}\right), \\
& \frac{\partial c_{t}}{\partial K_{t}}<0 \forall t \Leftrightarrow \min _{t}\left[\theta_{2}+\frac{1}{2}\left(\frac{\theta_{5} A_{t}^{2}+\boldsymbol{a}_{t}^{\top} \boldsymbol{\Theta}_{6} \boldsymbol{a}_{6}+\overline{\boldsymbol{Y}}_{t}^{\top} \boldsymbol{\Theta}_{7} \boldsymbol{Y}}{K_{t}^{2}}\right)\right]>\max _{t}\left(\frac{\alpha_{2}+\boldsymbol{\alpha}_{3}^{\top} \boldsymbol{w}_{t}}{\sqrt{\beta(\boldsymbol{w})}}\right), \\
& \frac{\partial c_{t}}{\partial \boldsymbol{a}_{t}}<\mathbf{0} \forall t \Leftrightarrow \boldsymbol{\theta}_{3}>\max _{t}\left(\frac{\boldsymbol{\Theta}_{6} \boldsymbol{a}_{6}}{K_{t}}\right), \\
& \frac{\partial c_{t}}{\partial \overline{\boldsymbol{Y}}_{t}}>\mathbf{0} \forall t \Leftrightarrow \boldsymbol{\theta}_{4}+\min _{t}\left(\frac{\boldsymbol{\Theta}_{7} \overline{\boldsymbol{Y}}_{t}}{K_{t}}\right)>0 .
\end{aligned}
$$

These can be imposed iteratively in estimation if necessary (LaFrance 1991). In this chapter, we checked for the monotonicity conditions at each data point given the parameter estimates obtained without imposing monotonicity.

Also, given that $K_{t}>0$, the implied curvature conditions are that the matrix

$$
\left[\begin{array}{cccc}
\theta_{5} K_{t}^{2} & -\theta_{5} A_{t} K_{t} & \mathbf{0}_{n_{y}}^{\top} & \mathbf{0}_{n_{y}}^{\top} \\
-\theta_{5} A_{t} K_{t} & \left(\theta_{5} A_{t}^{2}+\boldsymbol{a}_{t}^{\top} \boldsymbol{\Theta}_{6} \boldsymbol{a}_{6}+\overline{\boldsymbol{Y}}_{t}^{\top} \boldsymbol{\Theta}_{7} \overline{\boldsymbol{Y}}_{t}\right) & -K_{t} \boldsymbol{a}_{t}^{\top} \boldsymbol{\Theta}_{6} & -K_{t} \overline{\boldsymbol{Y}}_{t}^{\top} \boldsymbol{\Theta}_{7} \\
\mathbf{0}_{n_{y}} & -K_{t} \boldsymbol{\Theta}_{6} a_{6} & \boldsymbol{K}_{t}^{2} \boldsymbol{\Theta}_{6} & \mathbf{0}_{n_{y} \times n_{y}} \\
\mathbf{0}_{n_{y}} & -K_{t} \boldsymbol{\Theta}_{7} \overline{\boldsymbol{Y}}_{7} & \mathbf{0}_{n_{y} \times n_{y}} & K_{t}^{2} \boldsymbol{\Theta}_{7}
\end{array}\right]
$$

is positive semidefinite. This can be imposed during estimation with the Choleski factors, $\boldsymbol{\Theta}_{6}=\boldsymbol{L}_{6} \boldsymbol{L}_{6}^{\top}$ and $\boldsymbol{\Theta}_{7}=\boldsymbol{L}_{7} \boldsymbol{L}_{7}^{\top}$ with $\boldsymbol{L}_{6}$ and $\boldsymbol{L}_{7}$ lower triangular Choleski factors for $\boldsymbol{\Theta}_{6}$ and $\boldsymbol{\Theta}_{7}$, respectively, and the inequality $\theta_{5}>0$. In this chapter, only the matrix $\boldsymbol{\Theta}_{7}=\boldsymbol{L}_{7} \boldsymbol{L}_{7}^{\top}$ is estimated as part of the arbitrage conditions. 


\section{Appendix D}

\section{Specification Errors and Parameter Stability Tests}

Many diagnostic procedures for testing parameter stability and model specification errors have been developed. Few are designed for large systems of nonlinear simultaneous equations in small samples. These properties preclude using recursive-forecast residuals or Chow tests based on sequential sample splits to analyze specification errors or nonconstant parameters (Brown, Durbin, and Evans 1975; Harvey 1990, 1993; Hendry 1995). It is desirable to test whether the data are consistent with the model specification and constant parameters. LaFrance (2008) derived a set of specification and parameter stability diagnostics for this class of problems. These test statistics rely on the estimated in-sample residuals and have power against a range of alternatives, including nonconstant parameters and specification errors. The purpose of this section is to discuss briefly the main ideas that underpin this class of test statistics.

If the model is stationary and the errors are innovations, then consistent estimates of the model parameters can be found in any number of ways. Given consistent parameter estimates, the estimated errors converge in probability (and, therefore, in distribution) to the true errors, $\hat{\varepsilon}_{t} \stackrel{P}{\rightarrow} \varepsilon_{t}$. Therefore, for each $i=1, \ldots, n_{x}-1$, by the central limit theorem for stationary Martingale differences, we have

$$
\frac{1}{\sqrt{T} \sigma_{i}} \sum_{t=1}^{T} \varepsilon_{i t} \stackrel{D}{\rightarrow} N(0,1)
$$

where $\sigma_{i}^{2}=E\left(\varepsilon_{i t}^{2}\right)$ is the variance of the residual for the $i^{t h}$ demand equation. Moreover, for any given proportion of the sample, uniformly in $z \in[0,1]$,

$$
\frac{1}{\sqrt{T} \sigma_{i}} \sum_{t=1}^{[z T]} \varepsilon_{i t} \rightarrow N(0, z)
$$

where $[z T]$ is the largest integer that does not exceed $z T$. The variance is $z$ because we sum $[z T]$ independent terms each with variance $1 / T$. Multiplying equation (D1) by $z$ and subtracting from equation (D2) then gives

$$
\frac{1}{\sqrt{T} \sigma_{i}} \sum_{t=1}^{[z T]}\left(\varepsilon_{i t}-\bar{\varepsilon}_{i}\right) \stackrel{D}{\rightarrow} W(z)-z W(1) \equiv B(z),
$$

where $W(z)$ is a standard Brownian motion on the unit interval, with $W(z) \sim N(0, z)$, and $B(z)$ is a standard Brownian bridge, or tied Brownian motion. For all $z \in[0,1], B(z)$ has an asymptotic Gaussian distribution, with mean zero and standard deviation $[z(1-z)]^{1 / 2}$ (Bhattacharya and Waymire 1990). For a given $z$ - that is, to test for a break point in the model at a fixed 
and known date - an asymptotic 95 percent confidence interval for $B(z)$ is $\pm 1.96[z(1-z)]^{1 / 2}$. To check for an unknown break point, a statistic based on the supremum norm,

$$
Q_{T}=\sup _{z \in[0,1]}\left|B_{t}(z)\right|
$$

has an asymptotic 5 percent critical value of 1.36 (Ploberger and Krämer 1992).

We can use consistently estimated residuals and consistently estimated standard errors to obtain sample analogues to these asymptotic Brownian bridges. This gives

$$
B_{i T}(z) \equiv \frac{1}{\sqrt{T} \hat{\sigma}_{i}} \sum_{t=1}^{[z T]}\left(\hat{\varepsilon}_{i t}-\overline{\hat{\varepsilon}}_{i}\right) \stackrel{D}{\rightarrow} B(z),
$$

also uniformly in $z \in[0,1]$ so long as the model specification is correct and the parameters are constant across time periods. This statistic is a single equation first-order specification/parameter stability statistic because it is based on the first-order moment conditions, $E\left(\varepsilon_{i t}\right)=0 \forall i$, . A systemwide first-order specification/parameter stability statistic can be defined by

$$
B_{T}(z) \equiv \frac{1}{\sqrt{T}} \sum_{t=1}^{[z T]}\left[\frac{1}{\sqrt{n_{x}}} \sum_{i=1}^{n_{q}}\left(\hat{\xi}_{i t}-\overline{\hat{\xi}}\right)\right] \stackrel{D}{\rightarrow} B(z),
$$

where $\hat{\boldsymbol{\xi}}_{t}=\hat{\Sigma}^{-1 / 2} \hat{\varepsilon}_{t}$ is the $t^{\text {th }}$ estimated standardized error vector and $\overline{\hat{\xi}} \equiv$ $\sum_{t=1}^{T} \sum_{i=1}^{n_{x}} \hat{\xi}_{i i} / n_{x} T$.

Similar methods apply to second-order stationarity and parameter stability. We focus on systemwide statistics. Let $\boldsymbol{\Sigma}$ be factored into $\boldsymbol{L} \boldsymbol{L}^{\top}$, where $\boldsymbol{L}$ is lower triangular and nonsingular. Define the random vector $\boldsymbol{\xi}_{t}$ by $\boldsymbol{\varepsilon}_{t}=\boldsymbol{L} \boldsymbol{\xi}_{t}$. In addition to the preceding assumptions, add $\sup _{i, t} E\left(\varepsilon_{i t}^{4}\right)<\infty$. Estimate the within-period average sum of squared standardized residuals by

$$
\hat{v}_{t}=\frac{1}{n_{x}} \hat{\boldsymbol{\xi}}_{t}^{\top} \hat{\boldsymbol{\xi}}_{t}=\frac{1}{n_{x}} \hat{\boldsymbol{\varepsilon}}_{t}^{\top} \hat{\boldsymbol{\Sigma}}^{-1} \hat{\boldsymbol{\varepsilon}}_{t}
$$

where $\hat{\boldsymbol{\varepsilon}}_{t}$ is the vector of consistently estimated residuals in period $t$, and $\hat{\mathbf{\Sigma}}=\sum_{t=1}^{T} \hat{\boldsymbol{\varepsilon}}_{t} \hat{\boldsymbol{\varepsilon}}_{t}^{\top} / T$ is the associated consistently estimated error covariance matrix. The mean of the true $v_{t}$ is one for each $t$, and the martingale difference property of $\varepsilon_{t}$ is inherited by $v_{t}-1$. A consistent estimator of the asymptotic variance of $v_{t}$ is

$$
\hat{\sigma}_{v}^{2}=\frac{1}{T} \sum_{t=1}^{T}\left(\hat{v}_{t}^{2}-1\right)
$$

A systemwide second-order specification/parameter stability test statistic is obtained by calculating centered and standardized partial sums of $\hat{v}_{t}$, 


$$
B_{T}(z)=\frac{1}{\sqrt{T} \hat{\sigma}_{v}} \cdot \sum_{t=1}^{[z T]}\left(\hat{v}_{t}-1\right) \underset{T \rightarrow \infty}{\stackrel{D}{\longrightarrow}} B(z),
$$

uniformly in $z \in[0,1]$, where the limiting distribution on the far right follows from the identity $\widehat{\hat{v}} \equiv \sum_{t=1}^{T} \hat{v}_{t} / T \equiv 1$.

\section{References}

Akridge, J. T., and T. W. Hertel. 1986. "Multiproduct Cost Relationships for Retail Fertilizer Plants." American Journal of Agricultural Economics 68:928-38.

Babcock, B. A., and D. Hennessy. 1996. "Input Demand under Yield and Revenue Insurance." American Journal of Agricultural Economics 78:416-27.

Ball, E., R. Cavazos, J. T. LaFrance, R. Pope, and J. Tack. 2010. "Aggregation and Arbitrage in Joint Production." In The Economic Impact of Public Support to Agriculture, edited by E. Ball, R. Fanfani, and L. Gutierrez, 309-28. New York: Springer.

Ball, V. E., C. Hallahan, and R. Nehring. 2004. "Convergence of Productivity: An Analysis of the Catch-up Hypothesis within a Panel of States." American Journal of Agricultural Economics 86:1315-21.

Banks, J., R. Blundell, and A. Lewbel. 1997. "Quadratic Engel Curves and Consumer Demand." Review of Economics and Statistics 79:527-39.

Bellman, R., and S. Dreyfus. 1962. Applied Dynamic Programming. Princeton, NJ: Princeton University Press.

Bhattacharya, R. N., and E. C. Waymire. 1990. Stochastic Processes with Applications. New York: Wiley.

Blackorby, C., D. Primont, and R. Russell. 1977. "Dual Price and Quantity Aggregation." Journal of Economic Theory 14:130-48.

-1978. Separability and Functional Structure: Theory and Economic Applications. New York: American Elsevier/North-Holland.

Blundell, R. 1988. "Consumer Behavior: Theory and Empirical Evidence-A Survey" Economic Journal 98:16-65.

Brown, R. L., J. Durbin, and J. M. Evans. 1975. Techniques for Testing the Constancy of Regression Relationships over Time. Journal of the Royal Statistical Society Series B 37:149-192.

Chambers, R. G. 1984. "A Note on Separability of the Indirect Production Function and Measures of Substitution." Southern Economic Journal 4:1189-91.

1989. "Insurability and Moral Hazard in Agricultural Insurance Markets." American Journal of Agricultural Economics 71:604-16.

Chambers, R. G., and R. D. Pope. 1991. "Testing for Consistent Aggregation." American Journal of Agricultural Economics 73:808-18.

1994. "A Virtually Ideal Production System: Specifying and Estimating the VIPS Model.” American Journal of Agricultural Economics 76:105-13.

Chambers, R. G., and J. Quiggin. 2000. Uncertainty, Production, Choice and Agency: The State-Contingent Approach. Cambridge, UK: Cambridge University Press.

Chavas, J. P. 2008. "A Cost Approach to Economic Analysis under State-Contingent Production Uncertainty." American Journal of Agricultural Economics 90: 435-66.

Chavas J. P., and M. Holt. 1996. "Economic Behavior under Uncertainty: A Joint 
Analysis of Risk Preferences and Technology." Review of Economics and Statistics 78:329-35.

Deaton, A., and J. Muellbauer. 1980. "An Almost Ideal Demand System." American Economic Review 70:312-26.

Diewert, W. 1978. "Hicks' Aggregation Theorem and the Existence of a Real ValueAdded Function." In Production Economics: A Dual Approach to Theory and Applications. Vol. 2, edited by M. Fuss and D. McFadden, 17-51. New York: North-Holland.

Diewert, W. E., and T. J. Wales. 1987. "Flexible Functional Forms and Global Curvature Conditions." Econometrica 55:43-68.

- 1988. "Normalized Quadratic Systems of Consumer Demand Functions." Journal of Business and Economic Statistics 6:303-12.

Epstein, L. G. 1975. "A Disaggregate Analysis of Consumer Choice under Uncertainty.” Econometrica 47:877-92.

_. 1982. "Integrability of Incomplete Systems of Demand Functions." Review of Economic Studies 49:411-25.

Färe, R., and D. Primont. 1995. Multi-Output Production and Duality: Theory and Applications. Boston: Kluwer-Nijhoff.

Gardner, B. L., and R. A. Kramer. 1986. "Experience with Crop Insurance Programs in the United States." In Crop Insurance for Agricultural Development: Issues and Experience, edited by P. Hazell, C. Pomereda, and A. Valdes, 195-222. Baltimore: Johns Hopkins University Press.

Goldman, S. M., and H. Uzawa. 1964. "A Note on Separability in Demand Analysis." Econometrica 32:387-98.

Goodwin, B. K., and V. H. Smith. 1999. "An Ex-Post Evaluation of the Conservation Reserve, Federal Crop Insurance, and other Government Programs: Program Participation and Soil Erosion." Journal of Agricultural and Resource Economics 28 (2): 201-16.

Gorman, W. M. 1953. "Community Preference Fields.” Econometrica 21:63-80.

_. 1961. "On a Class of Preference Fields." Metroeconomica 13:53-6.

. 1981. "Some Engel Curves." In Essays in Honour of Sir Richard Stone, edited by A. Deaton, 7-29. Cambridge, UK: Cambridge University Press.

Hall, R. E. 1973. "The Specification of Technology with Several Kinds of Output." Journal of Political Economy 81:878-92.

Hansen, L. P., and K. J. Singleton. 1983. "Stochastic Consumption, Risk Aversion, and the Temporal Behavior of Asset Returns. Journal of Political Economy 91: 249-65.

Harvey, A. C. 1990. The Econometric Analysis of Time Series. Cambridge, MA: MIT Press.

- 1993. Time Series Models. 2nd ed. Cambridge, MA: MIT Press.

Hausman, J. 1981. "Exact Consumer's Surplus and Deadweight Loss." American Economic Review 71:662-76.

Hendry, D. F. 1995. Dynamic Econometrics. Oxford, UK: Oxford University Press.

Hoppe, R. A., and D. E. Banker. 2006. Structure and Finances of U.S. Farms: 2005 Family Farm Report. Economic Information Bulletin no. EIB-12. Washington, DC: Economic Research Service, U.S. Department of Agriculture.

Horowitz, J., and E. Lichtenberg. 1994. "Risk Reducing and Risk Increasing Effects of Pesticides." Journal of Agricultural Economics 45:82-9.

Howe, H., R. A. Pollak, and T. J. Wales. 1979. "Theory and Time Series Estimation of the Quadratic Expenditure System." Econometrica 47:1231-47.

Jerison, M. 1993. "Russell on Gorman's Engel Curves: A Correction.” Economics Letters 23:171-75. 
Jorgenson, D. W. 1990. "Consumer Behavior and the Measurement of Social Welfare." Econometrica 58:1007-40.

Jorgenson, D. W., L. J. Lau, and T. M. Stoker. 1980. "Welfare Comparisons under Exact Aggregation." American Economic Review 70:268-72.

1982. "The Transendental Logarithmic Model of Aggregate Consumer Behavior." In Advances in Econometrics, edited by R. L. Basmann and G. F. Rhodes, Jr., 97-238. Greenwich, CT: JAI Press.

Jorgenson, D. W., and D. T. Slesnick. 1984. "Aggregate Consumer Behavior and the Measurement of Inequality.” Review of Economic Studies 51:369-92. 1987. "Aggregate Consumer Behavior and Household Equivalence Scales." Journal of Business and Economic Statistics 5:219-32.

Just, R., D. Zilberman, and E. Hochman. 1983. "Estimation of Multicrop Production Functions." American Journal of Agricultural Economics 65:770-80.

Keeton, K., J. Skees, and J. Long. 1999. "The Potential Influence of Risk Management Programs on Cropping Decisions." Paper presented at the annual meeting of the American Agricultural Economics Association, Nashville, Tennessee.

Kohli, U. 1983. "Non-Joint Technologies." Review of Economic Studies 50:209-19.

LaFrance, J. T. 1985. "Linear Demand Functions in Theory and Practice." Journal of Economic Theory 37:147-66.

- 1986. "The Structure of Constant Elasticity Demand Models." American Journal of Agricultural Economics 68:543-52.

- 1990. "Incomplete Demand Systems and Semilogarithmic Demand Models." Australian Journal of Agricultural Economics 34:118-31.

—. 1991. "Consumer's Surplus versus Compensating Variation Revisited." American Journal of Agricultural Economics 73 (5): 1495-1507.

- 2004. "Integrability of the Linear Approximate Almost Ideal Demand System." Economic Letters 84:297-303.

- 2008. "The Structure of U.S. Food Demand." Journal of Econometrics $147: 336-49$.

LaFrance, J. T., T. K. M. Beatty, and R. D. Pope. 2006. "Gorman Engel Curves for Incomplete Demand Systems." In Exploring Frontiers in Applied Economics: Essays in Honor of Stanley R. Johnson, edited by M. T. Holt and J.-P. Chavas, 1-15. Berkeley, CA: Berkeley Electronic Press.

LaFrance, J. T., T. K. M. Beatty, R. D. Pope, and G. K. Agnew. 2002. "Information Theoretic Measures of the U.S. Variable Cost Distribution in Food Demand." Journal of Econometrics 107:235-57.

LaFrance, J. T., and W. M. Hanemann. 1989. "The Dual Structure of Incomplete Demand Systems." American Journal of Agricultural Economics 71:262-74.

LaFrance, J. T., and R. D. Pope. 2008. "Homogeneity and Supply." American Journal of Agricultural Economics 92:606-12.

_ 2009. "The Generalized Quadratic Expenditure System." In Contributions to Economic Analysis: Quantifying Consumer Preferences, edited by Daniel Slottje, 84-116. New York: Elsevier-Science.

2010. "Duality Theory for Variable Costs in Joint Production." American Journal of Agricultural Economics 92:755-62.

LaFrance, J. T., J. P. Shimshack, and S. Y. Wu. 2000. "Subsidized Crop Insurance and the Extensive Margin." Washington DC: USDA Economists Group, September.

. 2001. "The Environmental Impacts of Subsidized Crop Insurance." Proceedings of California Workshop on Environmental and Resource Economics, Santa Barbara, California.

. 2002. "Crop Insurance and the Extensive Margin.” Paper presented at World 
Congress of Environmental and Resource Economists (AERE/EAERE), Monterey, California.

2004. "Subsidized Crop Insurance and the Extensive Margin." In Risk Management in Agriculture. Rome, Italy: Instituto di Servizi per il Mercato Agricolo Alimentare.

Lau, L. 1972. "Profit Functions of Technologies with Multiple Input and Output." Review of Economics and Statistics 54:281-9.

1978. "Applications of Profit Functions." In Production Economics: A Dual Approach to Theory and Applications, edited by M. Fuss and D. McFadden, 134 216. Amsterdam: North-Holland.

1982. "A Note on the Fundamental Theorem of Exact Aggregation." Economics Letters 9:119-26.

Lewbel, A. 1987. "Characterizing Some Gorman Systems that Satisfy Consistent Aggregation." Econometrica 55:1451-59.

- 1988. "An Exactly Aggregable Trigonometric Engel Curve Demand System." Econometric Reviews 2:97-102.

_. 1989. "A Demand System Rank Theorem.” Econometrica 57:701-5.

289-300.

1990. "Full Rank Demand Systems." International Economic Review 31:

. 1991. "The Rank of Demand Systems: Theory and Nonparametric Estimation." Econometrica 59:711-30.

_. 2003. "A Rational Rank Four Demand System.” Journal of Applied Econometrics 18:127-35.

Lopez, R. 1983. "Structural Implications of a Class of Flexible Functional Forms for Profit Functions." International Economic Review 26:593-601.

Muellbauer, J. 1975. "Aggregation, Variable Cost Distribution and Consumer Demand." Review of Economic Studies 42:525-43.

_. 1976. "Community Preferences and the Representative Consumer." Econometrica 44:979-99.

Nelson, C. H., and E. T. Loehman. 1987. "Further toward a Theory of Agricultural Insurance." American Journal of Agricultural Economics 69:523-31.

Nerlove, M. 1958. The Dynamics of Supply: Estimation of Farmers' Response to Price. Baltimore: Johns Hopkins University Press.

Pope, R. D., and J.-P. Chavas. 1994. "Cost Functions under Production Uncertainty." American Journal of Agricultural Economics 76:196-204.

Pope, R. D., J. T. LaFrance, and R. E. Just. 2007. "Imperfect Price Deflation in Production Systems." American Journal of Agricultural Economics 89:738-54.

Ploberger, W., and W. Krämer. 1992. "The CUSUM Test with OLS Residuals." Econometrica 60:271-86.

Quiggin, J. 1992. "Some Observations on Insurance, Bankruptcy, and Input Demand." Journal of Economic Behavior and Organization 18:101-10.

Russell, T. 1983. "On a Theorem of Gorman.” Economic Letters 11:223-24.

1996. "Gorman Demand Systems and Lie Transformation Groups: A Reply.” Economic Letters 51:201-4.

Russell, T., and F. Farris. 1993. "The Geometric Structure of Some Systems of Demand Functions." Journal of Mathematical Economics 22:309-25.

1998. "Integrability, Gorman Systems, and the Lie Bracket Structure of the Real Line." Journal of Mathematical Economics 29:183-209.

Shumway, C. R. 1983. "Supply, Demand, and Technology in a Multiproduct Industry: Texas Field Crops." American Journal of Agricultural Economics 65:748-60.

Smith, V. H., and B. K. Goodwin. 1996. "Crop Insurance, Moral Hazard and Agricultural Chemical Use.” American Journal of Agricultural Economics 78:428-38. 
Soule, M., W. Nimon, and D. Mullarkey. 2000. "Risk Management and Environmental Outcomes: Framing the Issues." Paper presented at Crop Insurance, Land Use and the Environment Workshop (USDA/ERS), Washington D.C.

Turvey, C. G. 1992. "An Economic Analysis of Alternative Farm Revenue Insurance Policies." Canadian Journal of Agricultural Economics 40:403-26.

van Daal, J., and A. H. Q. M. Merkies. 1989. "A Note on the Quadratic Expenditure Model." Econometrica 57:1439-43.

Williams, J. R. 1988. "A Stochastic Dominance Analysis of Tillage and Crop Insurance Practices in a Semiarid Region." American Journal of Agricultural Economics 70:112-20.

Wright, B. D., and J. A. Hewitt. 1994. "All-Risk Crop Insurance: Lessons from Theory and Experience." In Economics of Agricultural Crop Insurance: Theory and Evidence, edited by D. L. Hueth and W. H. Furtan, 73-112. Natural Resource Management and Policy Series. Boston: Kluwer Academic.

Wu, J. 1999. "Crop Insurance, Acreage Decisions and Non-Point Source Pollution." American Journal of Agricultural Economics 81:305-20.

Young, C. E., R. D. Schnepf, J. R. Skees, and W. W. Lin. 2000. "Production and Price Impacts of U.S. Crop Insurance Subsidies: Some Preliminary Results.” Paper presented at Crop Insurance, Land Use and the Environment Workshop (USDA/ERS), Washington D.C. 